Article

\title{
Space of Quasi-Periodic Limit Functions and Its Applications
}

\author{
Rui Xie ${ }^{1, *} \mathbb{C}$, Zhinan $\mathrm{Xia}^{2}$ and Junwei Liu ${ }^{3}$ \\ 1 Department of Mathematics, Tianjin University of Commerce, Tianjin 300134, China \\ 2 Department of Applied Mathematics, Zhejiang University of Technology, \\ Hangzhou 310023, Zhejiang, China; xiazn299@zjut.edu.cn \\ 3 College of Science, Yanshan University, Qinhuangdao 066004, Hebei, China; liujunwei@ysu.edu.cn \\ * Correspondence: lxyxr@tjcu.edu.cn
}

Received: 23 October 2019; Accepted: 15 November 2019; Published: 19 November 2019

\begin{abstract}
We introduce a class consisting of what we call quasi-periodic limit functions and then establish the relation between quasi-periodic limit functions and asymptotically quasi-periodic functions. At last, these quasi-periodic limit functions are applied to study the existence of asymptotically quasi-periodic solutions of abstract Cauchy problems.
\end{abstract}

Keywords: quasi-periodic limit functions; asymptotically quasi-periodic functions; abstract Cauchy problems

MSC: 34C25, 34C27, 34A12

\section{Introduction}

The study of quasi-periodic functions may go back to P. Bohl in 1893 and E. Esclangon in 1904; see $[1,2]$. These quasi-periodic functions, which appeared in astronomical perturbation problems, are those that can be approximated uniformly on $\mathbb{R}$ by $\sum_{k=1}^{n} c_{k} A_{k}(t)$ with $c_{k} \in \mathbb{C}$ and $A_{k}(t)=$ $e^{i\left(m_{1} a_{1}+m_{2} a_{2}+\cdots+m_{n} a_{n}\right) t}$, where $m_{j} \in \mathbb{N}$ and $a_{j} \in \mathbb{R}$. It turns out that any quasi-periodic function can be obtained from a periodic function depending on several variables [3]. Let $f$ be a periodic function from $\mathbb{R}^{n}$ into $\mathbb{C}$. Regarding the variable $t_{i}$, if the periodic function $f\left(t_{1}, t_{2}, \cdots, t_{n}\right)$ has a period $2 \pi$, then $F(t)=f\left(\omega_{1} t, \omega_{2} t, \cdots, \omega_{n} t\right)$ with $\omega_{j} \in \mathbb{R} /\{0\}$ is quasi-periodic.

The application of quasi-periodic functions has been taken in several directions. One direction is the study of quasi-periodic solutions to nonlinear PDE. Up until now, the existence of quasi-periodic solutions of different kinds of nonlinear equations has been shown by the KAM (Kolmogorov-Arnold-Moser) theory or the C-W-B (Craig-Wayne-Bourgain) method. We briefly mention some recent work in this direction and refer the reader to [4-11] for some pioneering work. In [12], F. Giuliani focused on the generalized KdV equations and discussed the existence of Cantor families of quasi-periodic solutions, which extended the results in [13]. In [14], based on a modified KAM theorem, the existence of quasi-periodic response solutions of reversible systems that have Liouvillian frequencies was studied. Regarding the beam equations defined on compact Lie groups, in [15], the authors showed the existence of quasi-periodic solutions by the Nash-Moser iteration; meanwhile, based on the KAM theorem, in [16], Y. Wang proved the existence of quasi-periodic solutions to beam equations when the nonlinear term has the time and space variables. In [17], the authors discussed the Whitney smooth family of quasi-periodic solutions to beam equations. For other applications of quasi-periodic functions, we mention the work by Küpper and Yuan [18] on quasi-periodic solutions for differential equations with piecewise constant argument (see also, e.g., $[19,20])$. 
On the other hand, consider the inhomogeneous abstract Cauchy problem:

$$
\left\{\begin{array}{l}
x^{\prime}(t)=A x(t)+F(t), t \in \mathbb{R}^{+} ; \\
x(0)=x_{0} \in X
\end{array}\right.
$$

where $A$ is the infinitesimal generator of an exponentially stable $C_{0}$-semigroup $(T(t))_{t \geq 0}$, that is there exist $M>0$ and $r>0$ such that $\|T(t)\| \leq M e^{-r t}$ for all $t \in \mathbb{R}^{+}$. In order to study the existence of asymptotically periodic solutions of (1), the authors in [21] proposed the concept of the $\omega$-periodic limit function, which is a generalization of the asymptotically $\omega$-periodic function. Later, in [22], the concept of the squared mean periodic limit process was proposed and the application to stochastic differential equations was studied. The theory of $\omega$-periodic limit functions has the advantage of considering the asymptotical periodicity as a whole. For other applications of the $\omega$-periodic limit function, we refer the reader to [23].

It follows from the method in Theorem 4.4 in [21] that the solution of (1) is asymptotically $\omega$-periodic when the coefficient $F$ is the $\omega$-periodic limit. Moreover, if we assume $F=F_{1}+F_{2}, F_{1}$ is the $\omega_{1}$-periodic limit and $F_{2}$ is the $\omega_{2}$-periodic limit, then the solution of (1) is asymptotically quasi-periodic. Here, a question arises: To make sure the solution of (1) is asymptotically quasi-periodic, what kind of function can $F$ be?

Motivated by the above discussions, in the present paper, we propose a class of functions, and we call them quasi-periodic limit functions, which could be regarded as a generalization of (asymptotically) quasi-periodic functions. We will show that the quasi-periodic limit functions contribute to studying the existence of asymptotically quasi-periodic solutions of (1). The main results are Theorem 2 and Theorem 4. To show this, we develop a very general method. We believe the method in this paper could contribute to studying the existence of asymptotically quasi-periodic solutions of some kinds of equations, such as fractional differential equations.

This paper is arranged in three main sections.

In Section 2, we define the notion of quasi-periodic limit functions and study their properties. Especially, we discuss the relation between quasi-periodic limit functions and asymptotically quasi-periodic functions. In Section 3, these quasi-periodic limit functions are applied to study the existence of asymptotically quasi-periodic solutions of abstract Cauchy problems. In Section 4, we propose some related questions. We believe that the questions found here are of interest in the theory of asymptotical quasi-periodicity and that their answers would certainly help to develop this field.

\section{Space of Quasi-Periodic Limit Functions}

In this paper, we denote the interval $[0, \infty)$ by $\mathbb{R}^{+}$. Let $(X,\|\cdot\|)$ be a Banach space, and let $C_{b}\left(\mathbb{R}^{+} \times \mathbb{R}^{+}, X\right)$ be the space of bounded and continuous functions from $\mathbb{R}^{+} \times \mathbb{R}^{+}$into $X$, endowed with the uniform convergence norm $\|\cdot\|_{\infty}$. Assume that $\omega_{1}, \omega_{2}, \cdots, \omega_{n} \in \mathbb{R} . \omega_{1}, \omega_{2}, \cdots, \omega_{n}$ are called rationally independent if $k_{1} \omega_{1}+k_{2} \omega_{2}+\cdots+k_{n} \omega_{n} \neq 0$ for all $k_{1}, k_{2}, \cdots, k_{n} \in Q \backslash\{0\}$, where $Q$ is the set of all rational numbers. In this paper, we always assume $\omega_{1}, \omega_{2}, \cdots, \omega_{n}$ are rationally independent and $\omega_{1}, \omega_{2}, \cdots, \omega_{n}>0$. Let $f$ be a periodic function from $\mathbb{R}^{n}$ into $X$. If the periodic function $f\left(t_{1}, t_{2}, \cdots, t_{n}\right)$ in $t_{1}, t_{2}, \cdots, t_{n}$ with the same periodic $2 \pi$, then $F(t)=f\left(\frac{2 \pi}{\omega_{1}} t, \frac{2 \pi}{\omega_{2}} t, \cdots, \frac{2 \pi}{\omega_{n}} t\right)$ is said to be quasi-periodic. If we define $g\left(t_{1}, t_{2}, \cdots, t_{n}\right)=f\left(\frac{2 \pi}{\omega_{1}} t_{1}, \frac{2 \pi}{\omega_{2}} t_{2}, \cdots, \frac{2 \pi}{\omega_{n}} t_{n}\right)$, then: 


$$
\begin{aligned}
& g\left(t_{1}, t_{2}, \cdots, t_{k}+\omega_{k}, \cdots, t_{n}\right) \\
= & f\left(\frac{2 \pi}{\omega_{1}} t_{1}, \frac{2 \pi}{\omega_{2}} t_{2}, \cdots, \frac{2 \pi}{\omega_{k}}\left(t_{k}+\omega_{k}\right), \cdots, \frac{2 \pi}{\omega_{n}} t_{n}\right) \\
= & f\left(\frac{2 \pi}{\omega_{1}} t_{1}, \frac{2 \pi}{\omega_{2}} t_{2}, \cdots, \frac{2 \pi}{\omega_{k}} t_{k}+2 \pi, \cdots, \frac{2 \pi}{\omega_{n}} t_{n}\right) \\
= & f\left(\frac{2 \pi}{\omega_{1}} t_{1}, \frac{2 \pi}{\omega_{2}} t_{2}, \cdots, \frac{2 \pi}{\omega_{k}} t_{k}, \cdots, \frac{2 \pi}{\omega_{n}} t_{n}\right) \\
= & g\left(t_{1}, t_{2}, \cdots, t_{k}, \cdots, t_{n}\right)
\end{aligned}
$$

and we can define the same quasi-periodic function by $g$, that is $F(t)=g(t, t, \cdots, t)$. A continuous function $f: \mathbb{R}^{+} \rightarrow X$ is called asymptotically quasi-periodic if it admits a decomposition $f(t)=$ $g(t)+h(t)$, where $g: \mathbb{R} \rightarrow X$ is a quasi-periodic function and $h: \mathbb{R}^{+} \rightarrow X$ is a continuous function with $\lim _{t \rightarrow+\infty}\|h(t)\|=0$. Let $\omega>0$. A bounded continuous function $f: \mathbb{R}^{+} \rightarrow X$ is called the $\omega$-periodic limit if there is a function $g: \mathbb{R}^{+} \rightarrow X$ such that $\lim _{n \rightarrow \infty} f(t+n \omega)=g(t)$. The set of $\omega$-periodic limit functions will be denoted by $P_{\omega} L\left(\mathbb{R}^{+}, X\right)$.

For the sake of simplicity, we establish the concept by a function with two variables.

Definition 1. Let $f \in C_{b}\left(\mathbb{R}^{+} \times \mathbb{R}^{+}, X\right)$. If $g(t, s)=\lim _{n \rightarrow \infty} f\left(t+n \omega_{1}, s\right)$ and $h(t, s)=\lim _{n \rightarrow \infty} f(t, s+$ $\left.n \omega_{2}\right)$ are well defined for each pair $(t, s) \in \mathbb{R}^{+} \times \mathbb{R}^{+}$, where $n \in \mathbb{N}$, then $f$ is said to be the $\left(\omega_{1}, \omega_{2}\right)$-periodic limit.

Hypothesis 1 (H1). For each $t \in \mathbb{R}^{+}, \lim _{n \rightarrow \infty} f\left(t+n \omega_{1}, s\right)=g(t, s)$ uniformly for $s \in \mathbb{R}^{+}$.

Hypothesis 2 (H2). For each $s \in \mathbb{R}^{+}, \lim _{n \rightarrow \infty} f\left(t, s+n \omega_{2}\right)=h(t, s)$ uniformly for $t \in \mathbb{R}^{+}$.

Definition 2. Let $f$ be a $\left(\omega_{1}, \omega_{2}\right)$-periodic limit function. Assume $H 1$ and $H 2$ hold, then $F(t)=f(t, t)$ is said to be the $\left(\omega_{1}, \omega_{2}\right)$-quasi-periodic limit. The collection of all $\left(\omega_{1}, \omega_{2}\right)$-periodic limit functions that satisfies $H 1$ and $H 2$ (respectively, $\left(\omega_{1}, \omega_{2}\right)$-quasi-periodic limit functions) will be denoted by $P L_{\left(\omega_{1}, \omega_{2}\right)}\left(\mathbb{R}^{+} \times \mathbb{R}^{+}, X\right)$ $\left(Q P L_{\left(\omega_{1}, \omega_{2}\right)}\left(\mathbb{R}^{+}, X\right)\right)$.

Remark 1. The functions $g$ and $h$ in Definition 2 is measurable, but not necessarily continuous.

Now, we present some examples of $\left(\omega_{1}, \omega_{2}\right)$-quasi-periodic limit functions.

Example 1. Let $f(t, s)=f_{1}(t)+f_{2}(s)$, where $f_{1} \in P_{\omega_{1}} L\left(\mathbb{R}^{+}, X\right)$ and $f_{2} \in P_{\omega_{2}} L\left(\mathbb{R}^{+}, X\right)$. By the definition of the $\omega$-periodic limit function, we have $\lim _{n \rightarrow \infty} f_{1}\left(t+n \omega_{1}\right)=g_{1}(t)$ and $\lim _{n \rightarrow \infty} f_{2}\left(t+n \omega_{2}\right)=g_{2}(t)$. Let $g(t, s)=g_{1}(t)+f_{2}(s)$ and $h(t, s)=f_{1}(t)+g_{2}(s)$. Thus, for each $t \in \mathbb{R}^{+}, \lim _{n \rightarrow \infty} f\left(t+n \omega_{1}, s\right)=g(t, s)$ uniformly for $s \in \mathbb{R}^{+}$, and for each $s \in \mathbb{R}^{+}, \lim _{n \rightarrow \infty} f\left(t, s+n \omega_{2}\right)=h(t, s)$ uniformly for $t \in \mathbb{R}^{+}$. Then, $F(t)=f(t, t)=f_{1}(t)+f_{2}(t)$ is the $\left(\omega_{1}, \omega_{2}\right)$-quasi-periodic limit.

Example 2. Let $f(t, s)=f_{1}(t) f_{2}(s)$, where $f_{1} \in P_{\omega_{1}} L\left(\mathbb{R}^{+}, \mathbb{C}\right)$ and $f_{2} \in P_{\omega_{2}} L\left(\mathbb{R}^{+}, X\right)$. Then, $F(t)=$ $f(t, t)=f_{1}(t) f_{2}(t)$ is the $\left(\omega_{1}, \omega_{2}\right)$-quasi-periodic limit.

Example 3. For $n \geq 0$, define a function $f$ on $\left[n \omega_{1},(n+1) \omega_{1}\right] \times\left[m \omega_{2} \times(m+1) \omega_{2}\right]$ as follows: 


$$
f(t, s)= \begin{cases}1, & (t, s) \in\left[n \omega_{1}+\frac{\omega_{1}}{3}, n \omega_{1}+\frac{2 \omega_{1}}{3}\right] \times\left[m \omega_{2}+\frac{\omega_{2}}{3}, m \omega_{2}+\frac{2 \omega_{2}}{3}\right], \\ f_{n, m}^{1}(t, s), & (t, s) \in\left[n \omega_{1}+\frac{\omega_{1}}{3}-\frac{\omega_{1}}{n+3}, n \omega_{1}+\frac{\omega_{1}}{3}\right] \times\left[m \omega_{2}+\frac{\omega_{2}}{3}, m \omega_{2}+\frac{2 \omega_{2}}{3}\right], \\ f_{n, m}^{2}(t, s), & (t, s) \in\left[n \omega_{1}+\frac{2 \omega_{1}}{3}, n \omega_{1}+\frac{2 \omega_{1}}{3}+\frac{\omega_{1}}{n+3}\right] \times\left[m \omega_{2}+\frac{\omega_{2}}{3}, m \omega_{2}+\frac{2 \omega_{2}}{3}\right], \\ f_{n, m}^{3}(t, s), & (t, s) \in\left[n \omega_{1}+\frac{\omega_{1}}{3}, n \omega_{1}+\frac{2 \omega_{1}}{3}\right] \times\left[m \omega_{2}+\frac{\omega_{2}}{3}-\frac{\omega_{2}}{m+3}, m \omega_{2}+\frac{\omega_{2}}{3}\right], \\ f_{n, m}^{4}(t, s), & (t, s) \in\left[n \omega_{1}+\frac{\omega_{1}}{3}, n \omega_{1}+\frac{2 \omega_{1}}{3}\right] \times\left[m \omega_{2}+\frac{2 \omega_{2}}{3}, m \omega_{2}+\frac{2 \omega_{2}}{3}+\frac{\omega_{2}}{m+3}\right], \\ f_{n, m}^{5}(t, s), & (t, s) \in\left[n \omega_{1}+\frac{\omega_{1}}{3}-\frac{\omega_{1}}{n+3}, n \omega_{1}+\frac{\omega_{1}}{3}\right] \times\left[m \omega_{2}+\frac{\omega_{2}}{3}-\frac{\omega_{2}}{m+3}, m \omega_{2}+\frac{\omega_{2}}{3}\right], \\ f_{n, m}^{6}(t, s), & (t, s) \in\left[n \omega_{1}+\frac{2 \omega_{1}}{3}, n \omega_{1}+\frac{2 \omega_{1}}{3}+\frac{\omega_{1}}{n+3}\right] \times\left[m \omega_{2}+\frac{\omega_{2}}{3}-\frac{\omega_{2}}{m+3}, m \omega_{2}+\frac{\omega_{2}}{3}\right], \\ f_{n, m}^{7}(t, s), & (t, s) \in\left[n \omega_{1}+\frac{\omega_{1}}{3}-\frac{\omega_{1}}{n+3}, n \omega_{1}+\frac{\omega_{1}}{3}\right] \times\left[m \omega_{2}+\frac{2 \omega_{2}}{3}, m \omega_{2}+\frac{2 \omega_{2}}{3}+\frac{\omega_{2}}{m+3}\right], \\ f_{n, m}^{8}(t, s), & (t, s) \in\left[n \omega_{1}+\frac{2 \omega_{1}}{3}, n \omega_{1}+\frac{2 \omega_{1}}{3}+\frac{\omega_{1}}{n+3}\right] \times\left[m \omega_{2}+\frac{2 \omega_{2}}{3}, m \omega_{2}+\frac{2 \omega_{2}}{3}+\frac{\omega_{2}}{m+3}\right], \\ 0, & \text { otherwise, }\end{cases}
$$

where $f_{n, m}^{1}(t, s)=\frac{n+3}{\omega_{1}}\left[t-\left(n \omega_{1}+\frac{\omega_{1}}{3}-\frac{\omega_{1}}{n+3}\right)\right], f_{n, m}^{2}(t, s)=-\frac{n+3}{\omega_{1}}\left[t-\left(n \omega_{1}+\frac{2 \omega_{1}}{3}+\frac{\omega_{1}}{n+3}\right)\right]$, $f_{n, m}^{3}(t, s)=\frac{m+3}{\omega_{2}}\left[s-\left(m \omega_{2}+\frac{\omega_{2}}{3}-\frac{\omega_{2}}{m+3}\right)\right], \quad f_{n, m}^{4}(t, s)=-\frac{m+3}{\omega_{2}}\left[s-\left(m \omega_{2}+\frac{2 \omega_{2}}{3}+\frac{\omega_{2}}{m+3}\right)\right]$, $f_{n, m}^{5}(t, s)=\frac{\frac{n+3}{\omega_{1}}\left[t-\left(n \omega_{1}+\frac{\omega_{1}}{3}-\frac{\omega_{1}}{n+3}\right)\right]}{\frac{\omega_{2}}{m+3}}\left[s-\left(m \omega_{2}+\frac{\omega_{2}}{3}-\frac{\omega_{2}}{m+3}\right)\right], f_{n, m}^{6}(t, s)=-\frac{\frac{n+3}{\omega_{1}}\left[t-\left(n \omega_{1}+\frac{2 \omega_{1}}{3}+\frac{\omega_{1}}{n+3}\right)\right]}{\frac{\omega_{2}}{m+3}}[s-$ $\left.\left(m \omega_{2}+\frac{\omega_{2}}{3}-\frac{\omega_{2}}{m+3}\right)\right], f_{n, m}^{7}(t, s)=-\frac{\frac{n+3}{\omega_{1}}\left[t-\left(n \omega_{1}+\frac{\omega_{1}}{3}-\frac{\omega_{1}}{n+3}\right)\right]}{\frac{\omega_{2}}{m+3}}\left[s-\left(m \omega_{2}+\frac{2 \omega_{2}}{3}+\frac{\omega_{2}}{m+3}\right)\right], f_{n, m}^{8}(t, s)=$ $\frac{\frac{n+3}{\omega_{1}}\left[t-\left(n \omega_{1}+\frac{2 \omega_{1}}{3}+\frac{\omega_{1}}{n+3}\right)\right]}{\frac{\omega_{2}}{m+3}}\left[s-\left(m \omega_{2}+\frac{2 \omega_{2}}{3}+\frac{\omega_{2}}{m+3}\right)\right]$. The graph of the function $f$ in each rectangle $\left[n \omega_{1},(n+\right.$ 1) $\left.\omega_{1}\right] \times\left[m \omega_{1} \times(m+1) \omega_{2}\right](n \geq 1)$ consists of ten parts, and $f(t, s): \mathbb{R}^{+} \times \mathbb{R}^{+} \rightarrow[0,1]$ is continuous. If we define the function $g$ and $h$ on $\left[n \omega_{1},(n+1) \omega_{1}\right] \times\left[m \omega_{2} \times(m+1) \omega_{2}\right]$ by:

$$
g(t, s)= \begin{cases}1, & (t, s) \in\left[n \omega_{1}+\frac{\omega_{1}}{3}, n \omega_{1}+\frac{2 \omega_{1}}{3}\right] \times\left[m \omega_{2}+\frac{\omega_{2}}{3}, m \omega_{2}+\frac{2 \omega_{2}}{3}\right], \\ f_{n, m}^{3}(t, s), & (t, s) \in\left[n \omega_{1}+\frac{\omega_{1}}{3}, n \omega_{1}+\frac{2 \omega_{1}}{3}\right] \times\left[m \omega_{2}+\frac{\omega_{2}}{3}-\frac{\omega_{2}}{m+3}, m \omega_{2}+\frac{\omega_{2}}{3}\right], \\ f_{n, m}^{4}(t, s), & (t, s) \in\left[n \omega_{1}+\frac{\omega_{1}}{3}, n \omega_{1}+\frac{2 \omega_{1}}{3}\right] \times\left[m \omega_{2}+\frac{2 \omega_{2}}{3}, m \omega_{2}+\frac{2 \omega_{2}}{3}+\frac{\omega_{2}}{m+3}\right], \\ 0, & \text { otherwise }\end{cases}
$$

and:

$$
h(t, s)= \begin{cases}1, & (t, s) \in\left[n \omega_{1}+\frac{\omega_{1}}{3}, n \omega_{1}+\frac{2 \omega_{1}}{3}\right] \times\left[m \omega_{2}+\frac{\omega_{2}}{3}, m \omega_{2}+\frac{2 \omega_{2}}{3}\right] \\ f_{n, m}^{1}(t, s), & (t, s) \in\left[n \omega_{1}+\frac{\omega_{1}}{3}-\frac{\omega_{1}}{n+3}, n \omega_{1}+\frac{\omega_{1}}{3}\right] \times\left[m \omega_{2}+\frac{\omega_{2}}{3}, m \omega_{2}+\frac{2 \omega_{2}}{3}\right], \\ f_{n, m}^{2}(t, s), & (t, s) \in\left[n \omega_{1}+\frac{2 \omega_{1}}{3}, n \omega_{1}+\frac{2 \omega_{1}}{3}+\frac{\omega_{1}}{n+3}\right] \times\left[m \omega_{2}+\frac{\omega_{2}}{3}, m \omega_{2}+\frac{2 \omega_{2}}{3}\right], \\ 0, & \text { otherwise. }\end{cases}
$$

Then, for each $t \in \mathbb{R}^{+}, \lim _{n \rightarrow \infty} f\left(t+n \omega_{1}, s\right)=g(t, s)$ uniformly for $s \in \mathbb{R}^{+}$, and for each $s \in \mathbb{R}^{+}$, $\lim _{n \rightarrow \infty} f\left(t, s+n \omega_{2}\right)=h(t, s)$ uniformly for $t \in \mathbb{R}^{+}$. Thus, $F(t)=f(t, t)$ is the $\left(\omega_{1}, \omega_{2}\right)$-quasi-periodic limit.

Next, we present the following properties of $\left(\omega_{1}, \omega_{2}\right)$-quasi-periodic limit functions.

Proposition 1. Let $F, F_{1}$ and $F_{2}$ be the $\left(\omega_{1}, \omega_{2}\right)$-quasi-periodic limit. Assume $g(t, s)=\lim _{n \rightarrow \infty} f\left(t+n \omega_{1}, s\right)$, $h(t, s)=\lim _{n \rightarrow \infty} f\left(t, s+n \omega_{2}\right)$ are well defined for each pair $(t, s) \in \mathbb{R}^{+} \times \mathbb{R}^{+}$and $F(t)=f(t, t)$. Then, the following statements are true:

(1) $F_{1}+F_{2}$ is the $\left(\omega_{1}, \omega_{2}\right)$-quasi-periodic limit;

(2) $c F$ is the $\left(\omega_{1}, \omega_{2}\right)$-quasi-periodic limit for any $c \in \mathbb{C}$;

(3) $g\left(t+\omega_{1}, s\right)=g(t, s), h\left(t, s+\omega_{2}\right)=h(t, s)$ for each pair $(t, s) \in \mathbb{R}^{+} \times \mathbb{R}^{+}$;

(4) $g$ and $h$ are bounded on $\mathbb{R}^{+} \times \mathbb{R}^{+}$; moreover, $\|g\|_{\infty} \leq\|f\|_{\infty}$ and $\|h\|_{\infty} \leq\|f\|_{\infty}$; 
(5) $F$ is bounded on $\mathbb{R}^{+}$; moreover, $\|F\|_{\infty} \leq\|f\|_{\infty}$.

Proposition 2. Let $f$ be a $\left(\omega_{1}, \omega_{2}\right)$-periodic limit function.

(1) Assume H1 holds, then $k(t, s)=\lim _{n \rightarrow \infty} g\left(t, s+n \omega_{2}\right)$ is well defined for each pair $(t, s) \in \mathbb{R}^{+} \times \mathbb{R}^{+}$.

(2) Assume $H 2$ holds, then $\bar{k}(t, s)=\lim _{n \rightarrow \infty} h\left(t+n \omega_{1}, s\right)$ is well defined for each pair $(t, s) \in \mathbb{R}^{+} \times \mathbb{R}^{+}$.

(3) Assume H1 and $\mathrm{H} 2$ hold, then $k(t, s)=\bar{k}(t, s)$.

(4) Assume H1 and H2 hold, then $k\left(t+\omega_{1}, s\right)=k(t, s)=k\left(t, s+\omega_{2}\right)$.

Proof. (1) We only need to show that $\left\{g\left(t, s+n \omega_{2}\right)\right\}_{n \in \mathbb{N}}$ is a Cauchy sequence for each pair $(t, s) \in$ $\mathbb{R}^{+} \times \mathbb{R}^{+}$. Let $\varepsilon>0$. By Hypothesis of $\mathrm{H} 1$, for fixed $t \in \mathbb{R}^{+}$, there exists $N_{1} \in \mathbb{N}$ such that $\| f(t+$ $\left.p \omega_{1}, s\right)-g(t, s) \|<\frac{\varepsilon}{4}$ uniformly for $s \in \mathbb{R}^{+}$when $p \geq N_{1}$. For the above $t$, choose $p \geq N_{1}$, and fix $s \in \mathbb{R}^{+}$. By Definition 1 , there exists $N_{2} \in \mathbb{N}$ such that $\left\|f\left(t+p \omega_{1}, s+n \omega_{2}\right)-h\left(t+p \omega_{1}, s\right)\right\|<\frac{\varepsilon}{4}$ when $n \geq N_{2}$. Therefore,

$$
\begin{aligned}
& \left\|g\left(t, s+n \omega_{2}\right)-g\left(t, s+m \omega_{2}\right)\right\| \\
\leq & \left\|g\left(t, s+n \omega_{2}\right)-f\left(t+p \omega_{1}, s+n \omega_{2}\right)\right\|+\left\|f\left(t+p \omega_{1}, s+n \omega_{2}\right)-h\left(t+p \omega_{1}, s\right)\right\| \\
& +\left\|h\left(t+p \omega_{1}, s\right)-f\left(t+p \omega_{1}, s+m \omega_{2}\right)\right\|+\left\|f\left(t+p \omega_{1}, s+m \omega_{2}\right)-g\left(t, s+m \omega_{2}\right)\right\| \\
<\varepsilon &
\end{aligned}
$$

when $m, n \geq N_{2}$.

(2) In a similar way as (1), one can show (2).

(3) Let $\varepsilon>0$, and fix $(t, s) \in \mathbb{R}^{+} \times \mathbb{R}^{+}$. By Hypothesis $H 1$, there exists $N_{1} \in \mathbb{N}$ such that:

$$
\left\|f\left(t+n \omega_{1}, s^{\prime}\right)-g\left(t, s^{\prime}\right)\right\|<\frac{\varepsilon}{4}
$$

uniformly for $s^{\prime} \in \mathbb{R}^{+}$when $n \geq N_{1}$. By Hypothesis $H 2$, there exists $N_{2} \in \mathbb{N}$ such that:

$$
\left\|f\left(t^{\prime}, s+n \omega_{2}\right)-h\left(t^{\prime}, s\right)\right\|<\frac{\varepsilon}{4}
$$

uniformly for $t^{\prime} \in \mathbb{R}^{+}$when $n \geq N_{2}$. By the conclusion of (1), there exists $N_{3} \in \mathbb{N}$ such that:

$$
\left\|g\left(t, s+n \omega_{2}\right)-k(t, s)\right\|<\frac{\varepsilon}{4}
$$

when $n \geq N_{3}$. By the conclusion of (2), there exists $N_{4} \in \mathbb{N}$ such that:

$$
\left\|h\left(t+n \omega_{1}, s\right)-\bar{k}(t, s)\right\|<\frac{\varepsilon}{4}
$$

when $n \geq N_{4}$. Select $N_{5}=\max \left\{N_{1}, N_{2}, N_{3}, N_{4}\right\}$. (2), (3), (4), and (5) imply:

$$
\begin{aligned}
& \|k(t, s)-\bar{k}(t, s)\| \\
\leq & \left\|k(t, s)-g\left(t, s+N_{5} \omega_{2}\right)\right\|+\left\|g\left(t, s+N_{5} \omega_{2}\right)-f\left(t+N_{5} \omega_{1}, s+N_{5} \omega_{2}\right)\right\| \\
& +\left\|f\left(t+N_{5} \omega_{1}, s+N_{5} \omega_{2}\right)-h\left(t+N_{5} \omega_{1}, s\right)\right\|+\left\|h\left(t+N_{5} \omega_{1}, s\right)-\bar{k}(t, s)\right\| \\
<\varepsilon, &
\end{aligned}
$$

which shows $k(t, s)=\bar{k}(t, s)$.

(4) $k(t, s)=\lim _{n \rightarrow \infty} g\left(t, s+n \omega_{2}\right)=\lim _{n \rightarrow \infty} g\left(t+\omega_{1}, s+n \omega_{2}\right)=k\left(t+\omega_{1}, s\right)$. Similarly, $k(t, s)=$ $k\left(t, s+\omega_{2}\right)$.

Hypothesis 3 (H3). $f$ is uniformly continuous. 
Proposition 3. Let $f$ be a $\left(\omega_{1}, \omega_{2}\right)$-periodic limit function. Assume H3 holds, then $g$ and $h$ are uniformly continuous.

Proof. For $\varepsilon>0$ given, there exists $\delta>0$ such that $\left\|f(t, s)-f\left(t^{\prime}, s^{\prime}\right)\right\|<\frac{\varepsilon}{3}$ when $\left|t-t^{\prime}\right|<\delta$, $\left|s-s^{\prime}\right|<\delta$ for $t, t^{\prime}, s, s^{\prime} \in \mathbb{R}^{+}$. Choose $t, t^{\prime}, s, s^{\prime} \in \mathbb{R}^{+}$such that $\left|t-t^{\prime}\right|<\delta,\left|s-s^{\prime}\right|<\delta$, then there exists $N_{1} \in \mathbb{N}$ such that $\left\|g(t, s)-f\left(t+n \omega_{1}, s\right)\right\|<\frac{\varepsilon}{3}$ when $n \geq N_{1}$, and there exists $N_{2} \in \mathbb{N}$ such that $\left\|g\left(t^{\prime}, s^{\prime}\right)-f\left(t^{\prime}+n \omega_{1}, s^{\prime}\right)\right\|<\frac{\varepsilon}{3}$ when $n \geq N_{2}$. Choose $N_{3}=\max \left\{N_{1}, N_{2}\right\}$. Then, one has:

$$
\begin{aligned}
&\left\|g(t, s)-g\left(t^{\prime}, s^{\prime}\right)\right\| \\
& \leq\left\|g(t, s)-f\left(t+N_{3} \omega_{1}, s\right)\right\|+\left\|f\left(t+N_{3} \omega_{1}, s\right)-f\left(t^{\prime}+N_{3} \omega_{1}, s^{\prime}\right)\right\|+\left\|f\left(t^{\prime}+N_{3} \omega_{1}, s^{\prime}\right)-g\left(t^{\prime}, s^{\prime}\right)\right\| \\
&<\varepsilon .
\end{aligned}
$$

Therefore, $g$ is uniformly continuous. Similarly, $h$ is uniformly continuous.

In the following propositions, if $F$ is a $\left(\omega_{1}, \omega_{2}\right)$-quasi-periodic limit function, then $f, g, h$ are defined in Definition 2, and $k$ is defined in Proposition 2.

Proposition 4. Let $F$ be a $\left(\omega_{1}, \omega_{2}\right)$-quasi-periodic limit function. Assume $H 3$ holds, then $k$ is uniformly continuous.

Proof. Let $\varepsilon>0$. Since $f$ is uniformly continuous, $g$ is uniformly continuous by Proposition 3 . Therefore, there exists $\delta>0$ such that $\left\|g(t, s)-g\left(t^{\prime}, s^{\prime}\right)\right\|<\frac{\varepsilon}{3}$ when $\left|t-t^{\prime}\right|<\delta,\left|s-s^{\prime}\right|<\delta$ for $t, t^{\prime}, s, s^{\prime} \in \mathbb{R}^{+}$. Choose $t, t^{\prime}, s, s^{\prime} \in \mathbb{R}^{+}$such that $\left|t-t^{\prime}\right|<\delta,\left|s-s^{\prime}\right|<\delta$, then by Proposition 2 (1), there exists $N_{1} \in \mathbb{N}$ such that $\left\|k(t, s)-g\left(t, s+n \omega_{2}\right)\right\|<\frac{\varepsilon}{3}$ when $n \geq N_{1}$, and there exists $N_{2} \in \mathbb{N}$ such that $\left\|k\left(t^{\prime}, s^{\prime}\right)-g\left(t^{\prime}, s^{\prime}+n \omega_{2}\right)\right\|<\frac{\varepsilon}{3}$ when $n \geq N_{2}$. Choose $N_{3}=\max \left\{N_{1}, N_{2}\right\}$. Then, one has:

$$
\begin{aligned}
&\left\|k(t, s)-k\left(t^{\prime}, s^{\prime}\right)\right\| \\
& \leq\left\|k(t, s)-g\left(t, s+N_{3} \omega_{2}\right)\right\|+\left\|g\left(t, s+N_{3} \omega_{2}\right)-g\left(t^{\prime}, s^{\prime}+N_{3} \omega_{2}\right)\right\|+\left\|g\left(t^{\prime}, s^{\prime}+N_{3} \omega_{2}\right)-k\left(t^{\prime}, s^{\prime}\right)\right\| \\
&<\varepsilon .
\end{aligned}
$$

Thus, $k$ is uniformly continuous.

Proposition 5. Let $F$ be a $\left(\omega_{1}, \omega_{2}\right)$-quasi-periodic limit function. Assume H3 holds, and denote $r(t, s)=$ $f(t, s)-k(t, s)$, then $r \in C_{0}\left(\mathbb{R}^{+} \times \mathbb{R}^{+}, X\right)$, that is for any $\varepsilon>0$, there exists $M>0$ such that $\|r(t, s)\|<\varepsilon$ when $t>M, s>M$.

Proof. It is equivalent to show that for any $\varepsilon>0$, there exists $N \in \mathbb{N}$ such that $\| f\left(t+n \omega_{1}, s+m \omega_{2}\right)-$ $k\left(t+n \omega_{1}, s+m \omega_{2}\right) \|<\varepsilon$ uniformly for $t \in\left[0, \omega_{1}\right], s \in\left[0, \omega_{2}\right]$ when $n, m \geq N$. By Proposition 2 (4), one has $k(t, s)=k\left(t+n \omega_{1}, s+m \omega_{2}\right)$ for any $m, n \in \mathbb{N}$. Note that:

$$
\begin{aligned}
& f\left(t+n \omega_{1}, s+m \omega_{2}\right)-k\left(t+n \omega_{1}, s+m \omega_{2}\right) \\
= & f\left(t+n \omega_{1}, s+m \omega_{2}\right)-g\left(t, s+m \omega_{2}\right)+g\left(t, s+m \omega_{2}\right)-k(t, s) .
\end{aligned}
$$

Let $\varepsilon>0$. By Proposition 3 and Proposition $4, g$ and $k$ are uniformly continuous. Thus, there exists $\delta>0$ such that $\left\|f(t, s)-f\left(t^{\prime}, s^{\prime}\right)\right\|<\varepsilon,\left\|g(t, s)-g\left(t^{\prime}, s^{\prime}\right)\right\|<\varepsilon,\left\|k(t, s)-k\left(t^{\prime}, s^{\prime}\right)\right\|<\varepsilon$ when $\left|t-t^{\prime}\right|<\delta,\left|s-s^{\prime}\right|<\delta$ for $t, t^{\prime}, s, s^{\prime} \in \mathbb{R}^{+}$.

Divide $\left[0, \omega_{1}\right]$ into $p$ equal intervals such that $\frac{\omega_{1}}{p}<\delta, p \in \mathbb{N}$. Define $t_{0}=0, t_{1}=\frac{\omega_{1}}{p}, t_{2}=$ $\frac{2 \omega_{1}}{p}, \cdots, t_{p-1}=\frac{(p-1) \omega_{1}}{p}, t_{p}=\omega_{1}$. Then, there exists $N_{1} \in \mathbb{N}$ such that $\| f\left(t_{i}+n \omega_{1}, s+m \omega_{2}\right)-g\left(t_{i}, s+\right.$ $\left.m \omega_{2}\right) \|<\varepsilon$ uniformly for $s \in\left[0, \omega_{2}\right]$ and $m \in \mathbb{N}, i=0,1,2, \cdots, p$ when $n \geq N_{1}$. Choose any $t \in\left[0, \omega_{1}\right]$, one can pick $t_{i_{0}} \in\left\{t_{i}\right\}$ such that $\left|t-t_{i_{0}}\right|<\delta$. Then, one has: 


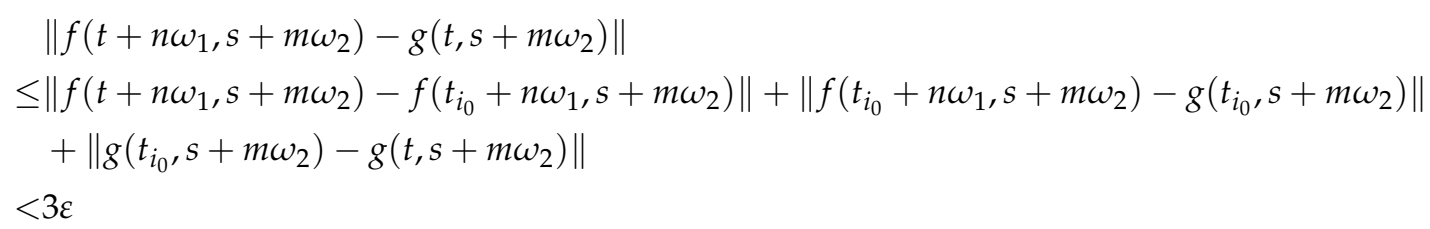

when $n \geq N_{1}$ uniformly for $s \in\left[0, \omega_{2}\right]$ and $m \in \mathbb{N}$.

Fix $t \in \mathbb{R}^{+}$. In a similar way, by dividing the interval $\left[0, \omega_{2}\right]$ and using the uniform continuousness of $g, k$, one can show there exists $N_{2} \in \mathbb{N}$ such that:

$$
\left\|g\left(t, s+m \omega_{2}\right)-k(t, s)\right\|<3 \varepsilon
$$

when $m \geq N_{2}$ uniformly for $s \in\left[0, \omega_{2}\right]$.

In a similar way again, by dividing the interval $\left[0, \omega_{1}\right]$ and using (6), one can show there exists $N_{3} \in \mathbb{N}$ such that $\left\|g\left(t, s+m \omega_{2}\right)-k(t, s)\right\|<5 \varepsilon$ when $m \geq N_{3}$ uniformly for $s \in\left[0, \omega_{2}\right]$ and $t \in$ $\left[0, \omega_{1}\right]$. Note that $g\left(t+n \omega_{1}, s\right)=g(t, s), k\left(t+n \omega_{1}, s\right)=k(t, s)$. Therefore, for any $t \in \mathbb{R}^{+}$, one has $\left\|g\left(t, s+m \omega_{2}\right)-k(t, s)\right\|<5 \varepsilon$ when $m \geq N_{3}$ uniformly for $s \in\left[0, \omega_{2}\right]$.

Choose $N_{4}=\max \left\{N_{1}, N_{3}\right\}$; one has:

$$
\begin{aligned}
& \left\|f\left(t+n \omega_{1}, s+m \omega_{2}\right)-k\left(t+n \omega_{1}, s+m \omega_{2}\right)\right\| \\
\leq & \left\|f\left(t+n \omega_{1}, s+m \omega_{2}\right)-g\left(t, s+m \omega_{2}\right)\right\|+\left\|g\left(t, s+m \omega_{2}\right)-k(t, s)\right\| \\
< & 8 \varepsilon
\end{aligned}
$$

when $n, m \geq N_{4}$ uniformly for $t \in\left[0, \omega_{1}\right]$ and $s \in\left[0, \omega_{2}\right]$.

Proposition 6. Let $F$ be a $\left(\omega_{1}, \omega_{2}\right)$-quasi-periodic limit function. Assume $H 3$ holds, then $F$ is asymptotically quasi-periodic.

Proof. Denote $K(t)=k(t, t), R(t)=r(t, t)$. By Proposition 5, $F(t)=K(t)+R(t), R \in C_{0}\left(\mathbb{R}^{+}, X\right)$. By Proposition $2(4)$ and Proposition $4, K$ is quasi-periodic. Therefore, $F$ is asymptotically quasi-periodic.

Let us introduce the following conditions.

Hypothesis $4(\mathrm{H} 4) . \lim _{n \rightarrow \infty} f\left(t+n \omega_{1}, s\right)=g(t, s)$ uniformly for $t \in \mathbb{R}^{+}$and $s \in \mathbb{R}^{+}$.

Hypothesis 5 (H5). $\lim _{n \rightarrow \infty} f\left(t, s+n \omega_{2}\right)=h(t, s)$ uniformly for $t \in \mathbb{R}^{+}$and $s \in \mathbb{R}^{+}$.

Proposition 7. Let $f$ be $a\left(\omega_{1}, \omega_{2}\right)$-periodic limit function. Assume H4 and H5 hold, then:

(1) $k(t, s)=\lim _{n \rightarrow \infty} g\left(t, s+n \omega_{2}\right)$ uniformly for $t \in \mathbb{R}^{+}$and $s \in \mathbb{R}^{+}$;

(2) $\bar{k}(t, s)=\lim _{n \rightarrow \infty} h\left(t+n \omega_{1}, s\right)$ uniformly for $t \in \mathbb{R}^{+}$and $s \in \mathbb{R}^{+}$;

(3) $k(t, s)=\bar{k}(t, s)$;

(4) $k\left(t+\omega_{1}, s\right)=k(t, s)=k\left(t, s+\omega_{2}\right)$.

Proof. (1) We only need to show that $\left\{g\left(t, s+n \omega_{2}\right)\right\}_{n \in \mathbb{N}}$ is a Cauchy sequence uniformly for $t \in \mathbb{R}^{+}$ and $s \in \mathbb{R}^{+}$. Let $\varepsilon>0$. By Hypothesis $\mathrm{H} 4$, there exists $N_{1} \in \mathbb{N}$ such that $\left\|f\left(t+p \omega_{1}, s\right)-g(t, s)\right\|<\frac{\varepsilon}{4}$ uniformly for $t \in \mathbb{R}^{+}$and $s \in \mathbb{R}^{+}$when $p \geq N_{1}$. By Hypothesis H5, there exists $N_{2} \in \mathbb{N}$ such that $\left\|f\left(t, s+n \omega_{2}\right)-h(t, s)\right\|<\frac{\varepsilon}{4}$ uniformly for $t \in \mathbb{R}^{+}$and $s \in \mathbb{R}^{+}$when $n \geq N_{2}$. 
Therefore,

$$
\begin{aligned}
& \left\|g\left(t, s+n \omega_{2}\right)-g\left(t, s+m \omega_{2}\right)\right\| \\
\leq & \left\|g\left(t, s+n \omega_{2}\right)-f\left(t+p \omega_{1}, s+n \omega_{2}\right)\right\|+\left\|f\left(t+p \omega_{1}, s+n \omega_{2}\right)-h\left(t+p \omega_{1}, s\right)\right\| \\
& +\left\|h\left(t+p \omega_{1}, s\right)-f\left(t+p \omega_{1}, s+m \omega_{2}\right)\right\|+\left\|f\left(t+p \omega_{1}, s+m \omega_{2}\right)-g\left(t, s+m \omega_{2}\right)\right\| \\
<\varepsilon &
\end{aligned}
$$

uniformly for $t \in \mathbb{R}^{+}$and $s \in \mathbb{R}^{+}$when $m, n \geq N_{2}$.

(2) In a similar way as (1), one can show (2).

(3) and (4) are the conclusions of Proposition 2 (3) (4).

Proposition 8. Let $F$ be a $\left(\omega_{1}, \omega_{2}\right)$-quasi-periodic limit function. Assume $H 4$ and $H 5$ hold, then $F$ is asymptotically quasi-periodic.

Proof. Denote $r(t, s)=f(t, s)-k(t, s)$; we first show that $r \in C_{0}\left(\mathbb{R}^{+} \times \mathbb{R}^{+}, X\right)$.

We only need to show that for any $\varepsilon>0$, there exists $N \in \mathbb{N}$ such that $\| f\left(t+n \omega_{1}, s+m \omega_{2}\right)-$ $k\left(t+n \omega_{1}, s+m \omega_{2}\right) \|<\varepsilon$ uniformly for $t \in\left[0, \omega_{1}\right], s \in\left[0, \omega_{2}\right]$ when $n, m \geq N$. By Proposition 7 (4), one has $k(t, s)=k\left(t+n \omega_{1}, s+m \omega_{2}\right)$ for any $m, n \in \mathbb{N}$.

By Hypothesis $\mathrm{H} 4$, there exists $N_{1} \in \mathbb{N}$ such that $\left\|f\left(t+n \omega_{1}, s\right)-g(t, s)\right\|<\frac{\varepsilon}{2}$ uniformly for $t \in \mathbb{R}^{+}$ and $s \in \mathbb{R}^{+}$when $n \geq N_{1}$. By Proposition 7, there exists $N_{2} \in \mathbb{N}$ such that $\left\|g\left(t, s+m \omega_{2}\right)-k(t, s)\right\|<\frac{\varepsilon}{2}$ uniformly for $t \in \mathbb{R}^{+}$and $s \in \mathbb{R}^{+}$when $m \geq N_{2}$. Choose $N=\max \left\{N_{1}, N_{2}\right\}$. Therefore,

$$
\begin{aligned}
& \left\|f\left(t+n \omega_{1}, s+m \omega_{2}\right)-k\left(t+n \omega_{1}, s+m \omega_{2}\right)\right\| \\
\leq & \left\|f\left(t+n \omega_{1}, s+m \omega_{2}\right)-g\left(t, s+m \omega_{2}\right)\right\|+\left\|g\left(t, s+m \omega_{2}\right)-k(t, s)\right\| \\
<\varepsilon &
\end{aligned}
$$

uniformly for $t \in\left[0, \omega_{1}\right], s \in\left[0, \omega_{2}\right]$ when $n, m \geq N$.

Next, we show that $k$ is continuous. Take any $t_{0}, s_{0} \in \mathbb{R}^{+}$. Since $f$ is continuous, there exists $\delta>0$ such that:

$$
\left\|f\left(t_{0}+N_{1} \omega_{1}, s_{0}+N_{2} \omega_{2}\right)-f\left(t^{\prime}+N_{1} \omega_{1}, s^{\prime}+N_{2} \omega_{2}\right)\right\|<\varepsilon
$$

when $\left|t_{0}-t^{\prime}\right|<\delta,\left|s_{0}-s^{\prime}\right|<\delta$. Therefore,

$$
\begin{aligned}
& \left\|k\left(t_{0}, s_{0}\right)-k\left(t^{\prime}, s^{\prime}\right)\right\| \\
= & \left\|k\left(t_{0}, s_{0}\right)-g\left(t_{0}, s_{0}+N_{2} \omega_{2}\right)\right\|+\left\|g\left(t_{0}, s_{0}+N_{2} \omega_{2}\right)-f\left(t_{0}+N_{1} \omega_{1}, s_{0}+N_{2} \omega_{2}\right)\right\| \\
& +\left\|f\left(t_{0}+N_{1} \omega_{1}, s_{0}+N_{2} \omega_{2}\right)-f\left(t^{\prime}+N_{1} \omega_{1}, s^{\prime}+N_{2} \omega_{2}\right)\right\| \\
& +\left\|f\left(t^{\prime}+N_{1} \omega_{1}, s^{\prime}+N_{2} \omega_{2}\right)-g\left(t^{\prime}, s^{\prime}+N_{2} \omega_{2}\right)\right\|+\left\|g\left(t^{\prime}, s^{\prime}+N_{2} \omega_{2}\right)-k\left(t^{\prime}, s^{\prime}\right)\right\| \\
< & 3 \varepsilon
\end{aligned}
$$

when $\left|t_{0}-t^{\prime}\right|<\delta,\left|s_{0}-s^{\prime}\right|<\delta$.

Denote $K(t)=k(t, t), R(t)=r(t, t)$. Then, $F(t)=K(t)+R(t), R \in C_{0}\left(\mathbb{R}^{+}, X\right)$, and $K$ is quasi-periodic. Therefore, $F$ is asymptotically quasi-periodic.

The collection of all $\left(\omega_{1}, \omega_{2}\right)$-periodic limit functions that satisfies $\mathrm{H} 4$ and $\mathrm{H} 5$ will be denoted by $A Q P_{\left(\omega_{1}, \omega_{2}\right)}\left(\mathbb{R}^{+} \times \mathbb{R}^{+}, X\right)$.

Theorem 1. $A Q P_{\left(\omega_{1}, \omega_{2}\right)}\left(\mathbb{R}^{+} \times \mathbb{R}^{+}, X\right)$ is a Banach space. 
Proof. Let $\left\{f_{k}\right\} \subset A Q P_{\left(\omega_{1}, \omega_{2}\right)}\left(\mathbb{R}^{+} \times \mathbb{R}^{+}, X\right)$ such that:

$$
\lim _{k \rightarrow \infty} f_{k}(t, s)=f(t, s)
$$

uniformly in $t, s \in \mathbb{R}^{+}$.

Since $\left\{f_{k}\right\} \subset A Q P_{\left(\omega_{1}, \omega_{2}\right)}\left(\mathbb{R}^{+} \times \mathbb{R}^{+}, X\right)$, for each $k \in \mathbb{N}$, one has:

$$
\lim _{n \rightarrow \infty} f_{k}\left(t+n \omega_{1}, s\right)=g_{k}(t, s)
$$

uniformly for $t \in \mathbb{R}^{+}$and $s \in \mathbb{R}^{+}$.

At the same time, for each $k \in \mathbb{N}$, one has:

$$
\lim _{n \rightarrow \infty} f_{k}\left(t, s+n \omega_{2}\right)=h_{k}(t, s)
$$

uniformly for $t \in \mathbb{R}^{+}$and $s \in \mathbb{R}^{+}$.

Then, the sequence of $\left\{g_{k}(t, s)\right\}$ is a Cauchy sequence in $X$ uniformly for $t \in \mathbb{R}^{+}$and $s \in \mathbb{R}^{+}$ because the following inequality:

$\left\|g_{k}(t, s)-g_{h}(t, s)\right\| \leq\left\|g_{k}(t, s)-f_{k}\left(t+n \omega_{1}, s\right)\right\|+\left\|f_{k}\left(t+n \omega_{1}, s\right)-f_{h}\left(t+n \omega_{1}, s\right)\right\|+\left\|f_{h}\left(t+n \omega_{1}, s\right)-g_{h}(t, s)\right\|$.

Thus, the sequence $\left\{g_{k}(t, s)\right\}$ converges to a function $g(t, s)$ uniformly for $t \in \mathbb{R}^{+}$and $s \in \mathbb{R}^{+}$.

Now, we only need to show:

$$
\lim _{n \rightarrow \infty} f\left(t+n \omega_{1}, s\right)=g(t, s)
$$

uniformly for $t \in \mathbb{R}^{+}$and $s \in \mathbb{R}^{+}$. However, we can get it from the following inequality.

$$
\left\|f\left(t+n \omega_{1}, s\right)-g(t, s)\right\| \leq\left\|f\left(t+n \omega_{1}, s\right)-f_{k}\left(t+n \omega_{1}, s\right)\right\|+\left\|f_{k}\left(t+n \omega_{1}, s\right)-g_{k}(t, s)\right\|+\left\|g_{k}(t, s)-g(t, s)\right\| .
$$

In a similar way, we can show that there exists a function $h$ such that:

$$
\lim _{n \rightarrow \infty} f\left(t, s+n \omega_{2}\right)=h(t, s)
$$

uniformly for $t \in \mathbb{R}^{+}$and $s \in \mathbb{R}^{+}$. Therefore, $f \in A Q P_{\left(\omega_{1}, \omega_{2}\right)}\left(\mathbb{R}^{+} \times \mathbb{R}^{+}, X\right)$.

\section{Existence of Asymptotically Quasi-Periodic Solutions of Abstract Cauchy Problems}

In this section, we first introduce the following definition:

Definition 3. A function $x \in C_{b}\left(\mathbb{R}^{+}, X\right)$ is said to be a mild solution of Problem (1) if:

$$
x(t)=T(t) x_{0}+\int_{0}^{t} T(t-s) F(s) d s, t \in \mathbb{R}^{+},
$$

where $(T(t))_{t \geq 0}$ is an exponentially stable $C_{0}$-semigroup.

Lemma 1. Let $F$ be a $\left(\omega_{1}, \omega_{2}\right)$-quasi-periodic limit function and $(T(t))_{t \geq 0}$ be an exponentially stable $C_{0}$-semigroup, then $U(t)=\int_{0}^{t} T(t-s) F(s) d s$ is asymptotically quasi-periodic.

Proof. By the definition of the $\left(\omega_{1}, \omega_{2}\right)$-quasi-periodic limit function, there exists a $f \in C_{b}\left(\mathbb{R}^{+} \times\right.$ $\left.\mathbb{R}^{+}, X\right)$ such that for each $t \in \mathbb{R}^{+}, \lim _{n \rightarrow \infty} f\left(t+n \omega_{1}, s\right)=g(t, s)$ uniformly for $s \in \mathbb{R}^{+}$, for each 
$s \in \mathbb{R}^{+}, \lim _{n \rightarrow \infty} f\left(t, s+n \omega_{2}\right)=h(t, s)$ uniformly for $t \in \mathbb{R}^{+}$and $F(t)=f(t, t)$. Assume $\|f(t, s)\| \leq K$ for $t, s \in \mathbb{R}^{+}$. Then, $\|g(t, s)\| \leq K$ and $\|h(t, s)\| \leq K$ for $t, s \in \mathbb{R}^{+}$.

Define:

$$
u(x, y)=\left\{\begin{array}{l}
\int_{0}^{x} T(x-s) f(s, s+y-x) d s, y \geq x, x, y \in \mathbb{R}^{+}, \\
\int_{0}^{y} T(y-s) f(s+x-y, s) d s, x \geq y, x, y \in \mathbb{R}^{+} .
\end{array}\right.
$$

Clearly, $u$ is continuous and bounded.

Note that $U(t)=u(t, t)=\int_{0}^{t} T(t-s) f(s, s) d s=\int_{0}^{t} T(t-s) F(s) d s$. By Proposition 8, we only need to show that $\mathrm{H} 4$ and $\mathrm{H} 5$ hold for $u$. Next, we only show that $\mathrm{H} 4$ holds for $u$ because the case for H5 is similar.

Define $f(t, s)=f(t, 0)$ and $g(t, s)=g(t, 0)$ when $s<0$. Then, we obtain $\lim _{n \rightarrow \infty} f\left(t+n \omega_{1}, s\right)=$ $\lim _{n \rightarrow \infty} f\left(t+n \omega_{1}, 0\right)=g(t, 0)=g(t, s)$ when $s<0$. Moreover, $\lim _{n \rightarrow \infty} f\left(t+n \omega_{1}, s\right)=g(t, s)$ uniformly for $s \in \mathbb{R}$. Note that $\int_{0}^{y} T(y-s) f(s+x-y, s) d s=\int_{x-y}^{x} T(x-s) f(s, s+y-x) d s$ when $x \geq y$. Then, one has:

$$
u(x, y)=\left\{\begin{array}{l}
\int_{0}^{x} T(x-s) f(s, s+y-x) d s, y \geq x, x, y \in \mathbb{R}^{+}, \\
\int_{0}^{x} T(x-s) f(s, s+y-x) d s-\int_{0}^{x-y} T(x-s) f(s, s+y-x) d s, x \geq y, x, y \in \mathbb{R}^{+} .
\end{array}\right.
$$

Denote $u_{1}(x, y)=\int_{0}^{x} T(x-s) f(s, s+y-x) d s$ and:

$$
u_{2}(x, y)=\left\{\begin{array}{l}
0, y \geq x, \\
\int_{0}^{x-y} T(x-s) f(s, s+y-x) d s, x \geq y,
\end{array}=\left\{\begin{array}{l}
0, y \geq x, \\
\int_{0}^{x-y} T(x-s) f(s, 0) d s, x \geq y .
\end{array}\right.\right.
$$

To show $\lim _{n \rightarrow \infty} u\left(x+n \omega_{1}, y\right)=v(x, y)$ uniformly for $x \in \mathbb{R}^{+}$and $y \in \mathbb{R}^{+}$, we only need to show $\lim _{n \rightarrow \infty} u_{1}\left(x+n \omega_{1}, y\right)=v_{1}(x, y)$ uniformly for $x \in \mathbb{R}^{+}$and $y \in \mathbb{R}^{+}$and $\lim _{n \rightarrow \infty} u_{2}\left(x+n \omega_{1}, y\right)=$ $v_{2}(x, y)$ uniformly for $x \in \mathbb{R}^{+}$and $y \in \mathbb{R}^{+}$.

Step 1. Let $x \in \mathbb{R}^{+}, y \in \mathbb{R}^{+}$.

$$
\begin{aligned}
u_{1}\left(x+n \omega_{1}, y\right) & =\int_{0}^{x+n \omega_{1}} T\left(x+n \omega_{1}-s\right) f\left(s, s+y-x-n \omega_{1}\right) d s \\
& =\int_{-n \omega_{1}}^{x} T(x-s) f\left(s+n \omega_{1}, s+y-x\right) d s \\
& =\int_{-n \omega_{1}}^{0} T(x-s) f\left(s+n \omega_{1}, s+y-x\right) d s+\int_{0}^{x} T(x-s) f\left(s+n \omega_{1}, s+y-x\right) d s \\
& =\int_{0}^{n \omega_{1}} T(x+s) f\left(n \omega_{1}-s, y-x-s\right) d s+\int_{0}^{x} T(x-s) f\left(s+n \omega_{1}, s+y-x\right) d s \\
& =I_{1}(x, y, n)+I_{2}(x, y, n) .
\end{aligned}
$$

We discuss the terms $I_{i}(x, y, n)(i=1,2)$ separately. First, we show that $I_{1}(x, y, n)$ is a Cauchy sequence in $X$ for each $x \in \mathbb{R}^{+}$and each $y \in \mathbb{R}^{+}$. 
For any $p \in \mathbb{N}, n \in \mathbb{N}$, one has:

$$
\begin{aligned}
& I_{1}(x, y, n+p)-I_{1}(x, y, n) \\
= & \int_{0}^{(n+p) \omega_{1}} T(x+s) f\left((n+p) \omega_{1}-s, y-x-s\right) d s \\
& -\int_{0}^{n \omega_{1}} T(x+s) f\left(n \omega_{1}-s, y-x-s\right) d s \\
= & \int_{n \omega_{1}}^{(n+p) \omega_{1}} T(x+s) f\left((n+p) \omega_{1}-s, y-x-s\right) d s \\
& +\int_{0}^{n \omega_{1}} T(x+s)\left[f\left((n+p) \omega_{1}-s, y-x-s\right)-f\left(n \omega_{1}-s, y-x-s\right)\right] d s \\
= & I_{3}(x, y, n, p)+I_{4}(x, y, n, p) .
\end{aligned}
$$

We see that:

$$
\begin{aligned}
\left\|I_{3}(x, y, n, p)\right\| & \leq \int_{n \omega_{1}}^{(n+p) \omega_{1}}\|T(x+s)\|\left\|f\left((n+p) \omega_{1}-s, y-x-s\right)\right\| d s \\
& \leq K M \int_{n \omega_{1}}^{(n+p) \omega_{1}} e^{-r(x+s)} d s \\
& \leq K M \int_{n \omega_{1}}^{\infty} e^{-r(x+s)} d s \\
& \leq \frac{K M}{r} e^{-r n \omega_{1}}
\end{aligned}
$$

Let $\varepsilon>0$. We can choose $N_{1} \in \mathbb{N}$ such that $\frac{K M}{r} e^{-r n \omega_{1}}<\varepsilon$ when $n \geq N_{1}$. Then, one gets $\left\|I_{3}(x, y, n, p)\right\|<\varepsilon$ when $n \geq N_{1}$ uniformly for $x \in \mathbb{R}^{+}$and $y \in \mathbb{R}^{+}$.

For $n \geq N_{1}$, we consider:

$$
\begin{aligned}
I_{4}(x, y, n, p)= & \int_{0}^{N_{1} \omega_{1}} T(x+s)\left[f\left((n+p) \omega_{1}-s, y-x-s\right)-f\left(n \omega_{1}-s, y-x-s\right)\right] d s \\
& +\int_{N_{1} \omega_{1}}^{n \omega_{1}} T(x+s)\left[f\left((n+p) \omega_{1}-s, y-x-s\right)-f\left(n \omega_{1}-s, y-x-s\right)\right] d s \\
= & I_{5}(x, y, n, p)+I_{6}(x, y, n, p) .
\end{aligned}
$$

Now, we estimate the term $I_{5}(x, y, n, p)$.

$$
\begin{aligned}
& \left\|I_{5}(x, y, n, p)\right\| \\
\leq & \int_{0}^{N_{1} \omega_{1}}\|T(x+s)\|\left\|f\left((n+p) \omega_{1}-s, y-x-s\right)-f\left(n \omega_{1}-s, y-x-s\right)\right\| d s \\
= & \int_{0}^{N_{1} \omega_{1}}\left\|T\left(x+N_{1} \omega_{1}-s\right)\right\| \| f\left(\left(n-N_{1}+p\right) \omega_{1}+s, s-N_{1} \omega_{1}+y-x\right) \\
& -f\left(\left(n-N_{1}\right) \omega_{1}+s, s-N_{1} \omega_{1}+y-x\right) \| d s \\
\leq & M \int_{0}^{N_{1} \omega_{1}} e^{-r\left(N_{1} \omega_{1}-s\right)}\left\|f\left(\left(n-N_{1}+p\right) \omega_{1}+s, s-N_{1} \omega_{1}+y-x\right)-g\left(s, s-N_{1} \omega_{1}+y-x\right)\right\| d s \\
+ & M \int_{0}^{N_{1} \omega_{1}} e^{-r\left(N_{1} \omega_{1}-s\right)}\left\|f\left(\left(n-N_{1}\right) \omega_{1}+s, s-N_{1} \omega_{1}+y-x\right)-g\left(s, s-N_{1} \omega_{1}+y-x\right)\right\| d s .
\end{aligned}
$$

For each $s \in\left[0, N_{1} \omega_{1}\right]$, we have:

$e^{-r\left(N_{1} \omega_{1}-s\right)}\left\|f\left(\left(n-N_{1}\right) \omega_{1}+s, s-N_{1} \omega_{1}+y-x\right)-g\left(s, s-N_{1} \omega_{1}+y-x\right)\right\| \leq 2 K e^{-r\left(N_{1} \omega_{1}-s\right)}$ 
and:

$$
\int_{0}^{N_{1} \omega_{1}} 2 K e^{-r\left(N_{1} \omega_{1}-s\right)} d s=\frac{2 K}{r}\left(1-e^{-r N_{1} \omega_{1}}\right) .
$$

Since $\lim _{n \rightarrow \infty} f\left(t+n \omega_{1}, s\right)=g(t, s)$ uniformly for $s \in \mathbb{R}$, for each $s \in\left[0, N_{1} \omega_{1}\right]$, one has:

$$
e^{-r\left(N_{1} \omega_{1}-s\right)}\left\|f\left(\left(n-N_{1}\right) \omega_{1}+s, s-N_{1} \omega_{1}+y-x\right)-g\left(s, s-N_{1} \omega_{1}+y-x\right)\right\| \rightarrow 0
$$

as $n \rightarrow \infty$. By Lebesgue's dominated convergence theorem, we obtain:

$$
\lim _{n \rightarrow \infty} \int_{0}^{N_{1} \omega_{1}} e^{-r\left(N_{1} \omega_{1}-s\right)}\left\|f\left(\left(n-N_{1}\right) \omega_{1}+s, s-N_{1} \omega_{1}+y-x\right)-g\left(s, s-N_{1} \omega_{1}+y-x\right)\right\| d s=0 .
$$

Since $\lim _{n \rightarrow \infty} f\left(t+n \omega_{1}, s\right)=g(t, s)$ uniformly for $s \in \mathbb{R},(8)$ holds uniformly for $x \in \mathbb{R}^{+}$and $y \in \mathbb{R}^{+}$. Moreover,

$$
\lim _{n \rightarrow \infty} \int_{0}^{N_{1} \omega_{1}} e^{-r\left(N_{1} \omega_{1}-s\right)}\left\|f\left(\left(n-N_{1}+p\right) \omega_{1}+s, s-N_{1} \omega_{1}+y-x\right)-g\left(s, s-N_{1} \omega_{1}+y-x\right)\right\| d s=0
$$

uniformly for $x \in \mathbb{R}^{+}$and $y \in \mathbb{R}^{+}$. Thus, we can select $N_{2} \in \mathbb{N}\left(N_{2}>N_{1}\right)$ such that $\left\|I_{5}(x, y, n, p)\right\|<\varepsilon$ when $n \geq N_{2}$ uniformly for $x \in \mathbb{R}^{+}$and $y \in \mathbb{R}^{+}$.

Next, we estimate the term $I_{6}(x, y, n, p)$.

$$
\begin{aligned}
\left\|I_{6}(x, y, n, p)\right\| & \leq \int_{N_{1} \omega_{1}}^{n \omega_{1}}\|T(x+s)\|\left\|f\left((n+p) \omega_{1}-s, y-x-s\right)-f\left(n \omega_{1}-s, y-x-s\right)\right\| d s \\
& \leq 2 K M \int_{N_{1} \omega_{1}}^{n \omega_{1}} e^{-r(x+s)} d s \\
& \leq 2 K M \int_{N_{1} \omega_{1}}^{\infty} e^{-r(x+s)} d s \\
& \leq \frac{2 K M}{r} e^{-r N_{1} \omega_{1}} \\
& <2 \varepsilon
\end{aligned}
$$

uniformly for $x \in \mathbb{R}^{+}$and $y \in \mathbb{R}^{+}$.

Thus, $\left\|I_{1}(x, y, n+p)-I_{1}(x, y, n)\right\| \leq\left\|I_{3}(x, y, n, p)\right\|+\left\|I_{5}(x, y, n, p)\right\|+\left\|I_{6}(x, y, n, p)\right\|<4 \varepsilon$ when $n \geq N_{2}$. This shows that $I_{1}(x, y, n)$ is a Cauchy sequence, and we denote $l_{1}(x, y)=\lim _{n \rightarrow \infty} I_{1}(x, y, n)$. Besides, from the proof, we also know that $\lim _{n \rightarrow \infty} I_{1}(x, y, n)=l_{1}(x, y)$ uniformly for $x \in \mathbb{R}^{+}$and $y \in \mathbb{R}^{+}$. 
Finally, we consider the term $I_{2}(x, y, n)$. Note that $\int_{0}^{x} T(x-s) g(s, s+y-x) d s$ is well defined for each $x \in \mathbb{R}^{+}$and $y \in \mathbb{R}^{+}$. For $m \omega_{1} \leq x<(m+1) \omega_{1}$, then one has:

$$
\begin{aligned}
& \left\|I_{2}(x, y, n)-\int_{0}^{x} T(x-s) g(s, s+y-x) d s\right\| \\
\leq & \int_{0}^{x}\|T(x-s)\|\left\|f\left(s+n \omega_{1}, s+y-x\right)-g(s, s+y-x)\right\| d s \\
\leq & M \int_{0}^{x} e^{-r(x-s)}\left\|f\left(s+n \omega_{1}, s+y-x\right)-g(s, s+y-x)\right\| d s \\
\leq & M \int_{0}^{m \omega_{1}} e^{-r(x-s)}\left\|f\left(s+n \omega_{1}, s+y-x\right)-g(s, s+y-x)\right\| d s \\
& +M \int_{m \omega_{1}}^{x}\left\|f\left(s+n \omega_{1}, s+y-x\right)-g(s, s+y-x)\right\| d s \\
\leq & M \sum_{k=0}^{m-1} \int_{k \omega_{1}}^{(k+1) \omega_{1}} e^{-r(x-s)}\left\|f\left(s+n \omega_{1}, s+y-x\right)-g(s, s+y-x)\right\| d s \\
& +M \int_{m \omega_{1}}^{(m+1) \omega_{1}}\left\|f\left(s+n \omega_{1}, s+y-x\right)-g(s, s+y-x)\right\| d s
\end{aligned}
$$

For each $t \in\left[0, \omega_{1}\right]$, we have $\left\|f\left(t+n \omega_{1}, s\right)-g(t, s)\right\| \rightarrow 0$ as $n \rightarrow \infty$ uniformly for $s \in \mathbb{R}$ and $\left\|f\left(t+n \omega_{1}, s\right)-g(t, s)\right\| \leq 2 K$. By Lebesgue's dominated convergence theorem, we obtain:

$$
\lim _{n \rightarrow \infty} \int_{0}^{\omega_{1}}\left\|f\left(t+n \omega_{1}, s\right)-g(t, s)\right\| d t=0
$$

uniformly for $s \in \mathbb{R}$. For $\varepsilon>0$ given, we select $N_{3} \in \mathbb{N}$ such that:

$$
\int_{0}^{\omega_{1}}\left\|f\left(t+n \omega_{1}, s\right)-g(t, s)\right\| d t<\varepsilon
$$

when $n \geq N_{3}$ uniformly for $s \in \mathbb{R}$. For any $i \in \mathbb{N}$, one has:

$$
\begin{aligned}
& \int_{i \omega_{1}}^{(i+1) \omega_{1}}\left\|f\left(t+n \omega_{1}, s\right)-g(t, s)\right\| d t \\
= & \int_{0}^{\omega_{1}}\left\|f\left(t+i \omega_{1}+n \omega_{1}, s\right)-g\left(t+i \omega_{1}, s\right)\right\| d t \\
= & \int_{0}^{\omega_{1}}\left\|f\left(t+i \omega_{1}+n \omega_{1}, s\right)-g(t, s)\right\| d t<\varepsilon
\end{aligned}
$$

when $n \geq N_{3}$ uniformly for $s \in \mathbb{R}$.

Therefore,

$$
\begin{aligned}
& \left\|I_{2}(x, y, n)-\int_{0}^{x} T(x-s) g(s, s+y-x) d s\right\| \\
\leq & M \sum_{k=0}^{m-1} e^{-r\left(x-(k+1) \omega_{1}\right)} \varepsilon+M \varepsilon \\
\leq & \left(\frac{1}{1-e^{-r \omega_{1}}}+1\right) M \varepsilon
\end{aligned}
$$

Hence, $\lim _{n \rightarrow \infty} I_{2}(x, y, n)=\int_{0}^{x} T(x-s) g(s, s+y-x) d s$ uniformly for $x \in \mathbb{R}^{+}$and $y \in \mathbb{R}^{+}$.

Therefore,

$$
\lim _{n \rightarrow \infty} u_{1}(x+n \omega, y)=\lim _{n \rightarrow \infty} I_{1}(x, y, n)+\lim _{n \rightarrow \infty} I_{2}(x, y, n)=l_{1}(x, y)+\int_{0}^{x} T(x-s) g(s, s+y-x) d s
$$

uniformly for $x \in \mathbb{R}^{+}$and $y \in \mathbb{R}^{+}$. 
Step 2. For any $x \in \mathbb{R}^{+}$. Firstly, let $y \in[0, x]$.

$$
\begin{aligned}
u_{2}\left(x+n \omega_{1}, y\right) & =\int_{0}^{x+n \omega_{1}-y} T\left(x+n \omega_{1}-s\right) f(s, 0) d s \\
& =\int_{-n \omega_{1}}^{x-y} T(x-s) f\left(s+n \omega_{1}, 0\right) d s \\
& =\int_{-n \omega_{1}}^{0} T(x-s) f\left(s+n \omega_{1}, 0\right) d s+\int_{0}^{x-y} T(x-s) f\left(s+n \omega_{1}, 0\right) d s \\
& =\int_{0}^{n \omega_{1}} T(x+s) f\left(n \omega_{1}-s, 0\right) d s+\int_{0}^{x-y} T(x-s) f\left(s+n \omega_{1}, 0\right) d s . \\
& =I_{7}(x, n)+I_{8}(x, y, n) .
\end{aligned}
$$

In a similar way as the case $I_{1}(x, y, n)$, we can show that $I_{7}(x, n)$ is a Cauchy sequence, and we denote $l_{2}(x)=\lim _{n \rightarrow \infty} I_{7}(x, n)$. Besides, we know $l_{2}(x)=\lim _{n \rightarrow \infty} I_{7}(x, n)$ uniformly for $x \in \mathbb{R}^{+}$.

Note that:

$$
\begin{aligned}
& \left\|I_{8}(x, y, n)-\int_{0}^{x-y} T(x-s) g(s, 0) d s\right\| \\
\leq & \int_{0}^{x-y}\|T(x-s)\|\left\|f\left(s+n \omega_{1}, 0\right)-g(s, 0)\right\| d s \\
\leq & \int_{0}^{x}\|T(x-s)\|\left\|f\left(s+n \omega_{1}, 0\right)-g(s, 0)\right\| d s \\
\leq & M \int_{0}^{x} e^{-r(x-s)}\left\|f\left(s+n \omega_{1}, 0\right)-g(s, 0)\right\| d s .
\end{aligned}
$$

Then, in a similar way as the case $I_{2}(x, y, n)$, we can show $\lim _{n \rightarrow \infty} I_{8}(x, y, n)=\int_{0}^{x-y} T(x-$ s) $g(s, 0) d s$ uniformly for $x \in \mathbb{R}^{+}$and $y \in[0, x]$.

Therefore,

$$
\lim _{n \rightarrow \infty} u_{2}(x+n \omega, y)=\lim _{n \rightarrow \infty} I_{7}(x, n)+\lim _{n \rightarrow \infty} I_{8}(x, y, n)=l_{2}(x)+\int_{0}^{x-y} T(x-s) g(s, 0) d s
$$

uniformly for $x \in \mathbb{R}^{+}$and $y \in[0, x]$.

Secondly, let $y>x$. Let $\varepsilon>0$, and choose $N_{1} \in \mathbb{N}$ such that $\frac{K M}{r} e^{-r n \omega_{1}}<\varepsilon$ when $n \geq N_{1}$. We now prove that $u_{2}\left(x+n \omega_{1}, y\right)$ is a Cauchy sequence in $X$ for each $y \in(x,+\infty)$. 
Case 1: $y-x \in\left(0, N_{1} \omega_{1}\right]$. Let $n>N_{1}, p \in \mathbb{N}$.

$$
\begin{aligned}
& u_{2}\left(x+(n+p) \omega_{1}, y\right)-u_{2}\left(x+n \omega_{1}, y\right) \\
= & \int_{0}^{x+(n+p) \omega_{1}-y} T\left(x+(n+p) \omega_{1}-s\right) f(s, 0) d s-\int_{0}^{x+n \omega_{1}-y} T\left(x+n \omega_{1}-s\right) f(s, 0) d s \\
= & \int_{-(n+p) \omega_{1}}^{x-y} T(x-s) f\left(s+(n+p) \omega_{1}, 0\right) d s-\int_{-n \omega_{1}}^{x-y} T(x-s) f\left(s+n \omega_{1}, 0\right) d s \\
= & \int_{y-x}^{(n+p) \omega_{1}} T(x+s) f\left((n+p) \omega_{1}-s, 0\right) d s-\int_{y-x}^{n \omega_{1}} T(x+s) f\left(n \omega_{1}-s, 0\right) d s \\
= & \int_{y-x}^{n \omega_{1}} T(x+s)\left[f\left((n+p) \omega_{1}-s, 0\right)-f\left(n \omega_{1}-s, 0\right)\right] d s+\int_{n \omega_{1}}^{(n+p) \omega_{1}} T(x+s) f\left((n+p) \omega_{1}-s, 0\right) d s \\
= & \int_{y-x}^{N_{1} \omega_{1}} T(x+s)\left[f\left((n+p) \omega_{1}-s, 0\right)-f\left(n \omega_{1}-s, 0\right)\right] d s \\
& +\int_{N_{1} \omega_{1}}^{n \omega_{1}} T(x+s)\left[f\left((n+p) \omega_{1}-s, 0\right)-f\left(n \omega_{1}-s, 0\right)\right] d s \\
& +\int_{n \omega_{1}}^{(n+p) \omega_{1}} T(x+s) f\left((n+p) \omega_{1}-s, 0\right) d s \\
= & I_{9}(x, y, n, p)+I_{10}(x, y, n, p)+I_{11}(x, y, n, p) .
\end{aligned}
$$

Now, we estimate the term $I_{9}(x, y, n, p)$.

$$
\begin{aligned}
\left\|I_{9}(x, y, n, p)\right\| \leq & \int_{0}^{N_{1} \omega_{1}} T(x+s)\left[f\left((n+p) \omega_{1}-s, 0\right)-f\left(n \omega_{1}-s, 0\right)\right] d s \\
= & \int_{0}^{N_{1} \omega_{1}} T\left(x+N_{1} \omega_{1}-s\right)\left[f\left(\left(n-N_{1}+p\right) \omega_{1}+s, 0\right)-f\left(\left(n-N_{1}\right) \omega_{1}+s, 0\right)\right] d s \\
\leq & M \int_{0}^{N_{1} \omega_{1}} e^{-r\left(N_{1} \omega_{1}-s\right)}\left\|f\left(\left(n-N_{1}+p\right) \omega_{1}+s, 0\right)-g(s, 0)\right\| d s \\
& +M \int_{0}^{N_{1} \omega_{1}} e^{-r\left(N_{1} \omega_{1}-s\right)}\left\|f\left(\left(n-N_{1}\right) \omega_{1}+s, 0\right)-g(s, 0)\right\| d s .
\end{aligned}
$$

By Lebesgue's dominated convergence theorem, there exists $N_{4} \in \mathbb{N}\left(N_{4}>N_{1}\right)$ such that $\left\|I_{9}(x, y, n, p)\right\|<\varepsilon$ when $n \geq N_{4}$ uniformly for $y-x \in\left(0, N_{1} \omega_{1}\right]$ and $x \in \mathbb{R}^{+}$. Since $\frac{K M}{r} e^{-r n \omega_{1}}<$ $\varepsilon\left(n \geq N_{1}\right)$, then we have:

$$
\begin{aligned}
\left\|I_{10}(x, y, n, p)\right\| & \leq 2 K M \int_{N_{1} \omega_{1}}^{n \omega_{1}} e^{-r(x+s)} d s \\
& \leq 2 K M \int_{N_{1} \omega_{1}}^{\infty} e^{-r(x+s)} d s \\
& \leq \frac{2 K M}{r} e^{-r N_{1} \omega_{1}} \\
& <2 \varepsilon
\end{aligned}
$$

and:

$$
\begin{aligned}
\left\|I_{11}(x, y, n, p)\right\| & \leq K M \int_{n \omega_{1}}^{(n+p) \omega_{1}} e^{-r(x+s)} d s \\
& \leq K M \int_{n \omega_{1}}^{\infty} e^{-r(x+s)} d s \\
& \leq \frac{K M}{r} e^{-r n \omega_{1}} \\
& <\varepsilon
\end{aligned}
$$


uniformly for $y-x \in\left(0, N_{1} \omega_{1}\right]$ and $x \in \mathbb{R}^{+}$.

Therefore, $\left\|u_{2}\left(x+(n+p) \omega_{1}, y\right)-u_{2}\left(x+n \omega_{1}, y\right)\right\|<4 \varepsilon$ when $n \geq N_{4}$ uniformly for $y-x \in$ $\left(0, N_{1} \omega_{1}\right]$ and $x \in \mathbb{R}^{+}$.

Case 2: $y-x \in\left(N_{1} \omega_{1}, N_{4} \omega_{1}\right]$. Note that $\frac{K M}{r} e^{-r n \omega_{1}}<\varepsilon\left(n \geq N_{1}\right)$.

$$
\begin{aligned}
& \left\|u_{2}\left(x+(n+p) \omega_{1}, y\right)-u_{2}\left(x+n \omega_{1}, y\right)\right\| \\
\leq & \left\|\int_{0}^{x+(n+p) \omega_{1}-y} T\left(x+(n+p) \omega_{1}-s\right) f(s, 0) d s\right\|+\left\|\int_{0}^{x+n \omega_{1}-y} T\left(x+n \omega_{1}-s\right) f(s, 0) d s\right\| \\
= & \left\|\int_{-(n+p) \omega_{1}}^{x-y} T(x-s) f\left(s+(n+p) \omega_{1}, 0\right) d s\right\|+\left\|\int_{-n \omega_{1}}^{x-y} T(x-s) f\left(s+n \omega_{1}, 0\right) d s\right\| \\
= & \left\|\int_{y-x}^{(n+p) \omega_{1}} T(x+s) f\left((n+p) \omega_{1}-s, 0\right) d s\right\|+\left\|\int_{y-x}^{n \omega_{1}} T(x+s) f\left(n \omega_{1}-s, 0\right) d s\right\| \\
\leq & K M \int_{y-x}^{(n+p) \omega_{1}} e^{-r(x+s)} d s+K M \int_{y-x}^{n \omega_{1}} e^{-r(x+s)} d s \\
\leq & K M \int_{y-x}^{\infty} e^{-r(x+s)} d s+K M \int_{y-x}^{\infty} e^{-r(x+s)} d s \\
\leq & \frac{K M}{r} e^{-r(y-x)}+\frac{K M}{r} e^{-r(y-x)} \\
< & 2 \varepsilon
\end{aligned}
$$

when $n \geq N_{4}$ uniformly for $y-x \in\left(N_{1} \omega_{1}, N_{4} \omega_{1}\right]$ and $x \in \mathbb{R}^{+}$.

Case 3: $y-x \in\left(N_{4} \omega_{1},+\infty\right)$. Consider $n \geq N_{4}$. Note that:

$$
u_{2}\left(x+n \omega_{1}, y\right)= \begin{cases}\int_{y-x}^{n \omega_{1}} T(x+s) f\left(n \omega_{1}-s, 0\right) d s, & y \leq x+n \omega_{1} \\ 0, & y \geq x+n \omega_{1}\end{cases}
$$

and:

$$
u_{2}\left(x+(n+p) \omega_{1}, y\right)= \begin{cases}\int_{y-x}^{(n+p) \omega_{1}} T(x+s) f\left((n+p) \omega_{1}-s, 0\right) d s, & y \leq x+(n+p) \omega_{1}, \\ 0, & y \geq x+(n+p) \omega_{1} .\end{cases}
$$

Then, one has:

$$
\begin{aligned}
&\left\|u_{2}\left(x+n \omega_{1}, y\right)\right\| \\
&=\left\|\int_{y-x}^{n \omega_{1}} T(x+s) f\left(n \omega_{1}-s, 0\right) d s\right\| \\
& \leq K M \int_{y-x}^{n \omega_{1}} e^{-r(x+s)} d s \\
& \leq K M \int_{y-x}^{\infty} e^{-r(x+s)} d s \\
& \leq \frac{K M}{r} e^{-r(y-x)} \\
&<\varepsilon
\end{aligned}
$$

when $y-x \in\left(N_{4} \omega_{1}, n \omega_{1}\right]$.

Besides, $\left\|u_{2}\left(x+n \omega_{1}, y\right)\right\|=0$ when $y-x \in\left(n \omega_{1},+\infty\right)$. Therefore, $\left\|u_{2}\left(x+n \omega_{1}, y\right)\right\|<\varepsilon$ when $y-x \in\left(N_{4} \omega_{1},+\infty\right)$. Similarly, $\left\|u_{2}\left(x+(n+p) \omega_{1}, y\right)\right\|<\varepsilon$ when $y-x \in\left(N_{4} \omega_{1},+\infty\right)$. Thus, 
$\left\|u_{2}\left(x+(n+p) \omega_{1}, y\right)-u_{2}\left(x+n \omega_{1}, y\right)\right\|<2 \varepsilon$ when $n \geq N_{4}$ uniformly for $y-x \in\left(N_{4} \omega_{1},+\infty\right)$ and $x \in \mathbb{R}^{+}$.

Therefore, $\left\|u_{2}\left(x+(n+p) \omega_{1}, y\right)-u_{2}\left(x+n \omega_{1}, y\right)\right\|<4 \varepsilon$ when $n \geq N_{4}$ uniformly for $y-x \in$ $(0,+\infty)$ and $x \in \mathbb{R}^{+}$. This shows that $u_{2}\left(x+n \omega_{1}, y\right)$ is a Cauchy sequence, and we denote $l_{3}(x, y)=$ $\lim _{n \rightarrow \infty} u_{2}\left(x+n \omega_{1}, y\right)$. Besides, from the proof, we also know that $\lim _{n \rightarrow \infty} u_{2}\left(x+n \omega_{1}, y\right)=l_{3}(x, y)$ uniformly for $y \in(x,+\infty)$ and $x \in \mathbb{R}^{+}$.

Therefore,

$$
\lim _{n \rightarrow \infty} u_{2}\left(x+n \omega_{1}, y\right)= \begin{cases}l_{2}(x)+\int_{0}^{x-y} T(x-s) g(s, 0) d s, & y \in[0, x], \\ l_{3}(x, y), & y \in(x,+\infty)\end{cases}
$$

uniformly for $x \in \mathbb{R}^{+}$and $y \in \mathbb{R}^{+}$.

This completes the proof.

Theorem 2. Let $F$ be a $\left(\omega_{1}, \omega_{2}\right)$-quasi-periodic limit function. Then, the mild solution of Problem (1) is asymptotically quasi-periodic.

Remark 2. Theorem 4.4 in [21] implies that the mild solution of Problem (1) is asymptotically quasi-periodic when $F=F_{1}+F_{2}$, where $F_{1} \in P_{\omega_{1}} L\left(\mathbb{R}^{+}, X\right), F_{2} \in P_{\omega_{2}} L\left(\mathbb{R}^{+}, X\right)$. However, the method in [21] is not available when $F=F_{1} F_{2}$, where $F_{1} \in P_{\omega_{1}} L\left(\mathbb{R}^{+}, \mathbb{C}\right), F_{2} \in P_{\omega_{2}} L\left(\mathbb{R}^{+}, X\right)$. Therefore, we propose the concept of the quasi-periodic limit function and develop the method to get a more general result.

Next, consider the following abstract Cauchy problem:

$$
\left\{\begin{array}{l}
x^{\prime}(t)=A x(t)+F(t, x(t)), t \in \mathbb{R}^{+} \\
x(0)=x_{0} \in X
\end{array}\right.
$$

where $A$ is the infinitesimal generator of an exponentially stable $C_{0}$-semigroup $(T(t))_{t \geq 0}$.

Let us introduce the following definition.

Definition 4. A joint continuous function $f: \mathbb{R}^{+} \times \mathbb{R}^{+} \times X \rightarrow X$ is said to be the $\left(\omega_{1}, \omega_{2}\right)$-periodic limit uniformly for $x$ in bounded subsets of $X$ if for every bounded subset $K$ of $X,\left\{f(t, s, x): t, s \in \mathbb{R}^{+}, x \in K\right\}$ is bounded, for each $t \in \mathbb{R}^{+} \lim _{n \rightarrow \infty} f\left(t+n \omega_{1}, s, x\right)=g(t, s, x)$ uniformly for $s \in \mathbb{R}^{+}$and $x \in K$, for each $s \in \mathbb{R}^{+} \lim _{n \rightarrow \infty} f\left(t, s+n \omega_{2}, x\right)=h(t, s, x)$ uniformly for $t \in \mathbb{R}^{+}$and $x \in K$. Denote by $P L_{\left(\omega_{1}, \omega_{2}\right)}\left(\mathbb{R}^{+} \times \mathbb{R}^{+} \times X, X\right)$ the set of all such functions. If we define $F(t, x)=f(t, t, x)$, then $F(t, x)$ is said to be the $\left(\omega_{1}, \omega_{2}\right)$-quasi-periodic limit uniformly for $x$ in bounded subsets of $X$.

The following is a composition theorem.

Theorem 3. Let $f: \mathbb{R}^{+} \times \mathbb{R}^{+} \times X \rightarrow X$ be the $\left(\omega_{1}, \omega_{2}\right)$-periodic limit uniformly for $x$ in bounded subsets of $X$, and assume that $f$ satisfies a Lipschitz condition in $x$ uniformly in $t, s \in \mathbb{R}^{+}$:

$$
\|f(t, s, x)-f(t, s, y)\| \leq L\|x-y\|
$$

for all $x, y \in X$ and $t, s \in \mathbb{R}^{+}$, where $L>0$. Let $\varphi \in P L_{\left(\omega_{1}, \omega_{2}\right)}\left(\mathbb{R}^{+} \times \mathbb{R}^{+}, X\right)$. The function $F: \mathbb{R}^{+} \times \mathbb{R}^{+} \rightarrow$ $X$ is defined by $F(t, s)=f(t, s, \varphi(t, s))$. Then, $F \in P L_{\left(\omega_{1}, \omega_{2}\right)}\left(\mathbb{R}^{+} \times \mathbb{R}^{+}, X\right)$.

Proof. Since $\varphi \in P L_{\left(\omega_{1}, \omega_{2}\right)}\left(\mathbb{R}^{+} \times \mathbb{R}^{+}, X\right)$, we have for each $t \in \mathbb{R}^{+}$:

$$
\lim _{n \rightarrow \infty} \varphi\left(t+n \omega_{1}, s\right)=\varphi_{g}(t, s)
$$

uniformly for $s \in \mathbb{R}^{+}$. 
Select a bounded subset $K$ of $X$ such that $\varphi(t, s), \varphi_{g}(t, s) \in K$ for $t, s \in \mathbb{R}^{+}$. Thus, $F(t, s)$ is bounded.

On the other side, one has for each $t \in \mathbb{R}^{+}$:

$$
\lim _{n \rightarrow \infty} f\left(t+n \omega_{1}, s, x\right)=g(t, s, x)
$$

uniformly for $s \in \mathbb{R}^{+}$and $x \in K$.

Let us consider the function $G: \mathbb{R}^{+} \times \mathbb{R}^{+} \rightarrow X$ defined by $G(t, s)=g\left(t, s, \varphi_{g}(t, s)\right)$. Note that for each $t \in \mathbb{R}^{+}$:

$$
\begin{aligned}
\left\|F\left(t+n \omega_{1}, s\right)-G(t, s)\right\| \leq & \left\|f\left(t+n \omega_{1}, s, \varphi\left(t+n \omega_{1}, s\right)\right)-f\left(t+n \omega_{1}, s, \varphi_{g}(t, s)\right)\right\| \\
& +\left\|f\left(t+n \omega_{1}, s, \varphi_{g}(t, s)\right)-g\left(t, s, \varphi_{g}(t, s)\right)\right\| \\
\leq & L\left\|\varphi\left(t+n \omega_{1}, s\right)-\varphi_{g}(t, s)\right\|+\left\|f\left(t+n \omega_{1}, s, \varphi_{g}(t, s)\right)-g\left(t, s, \varphi_{g}(t, s)\right)\right\|
\end{aligned}
$$

uniformly for $s \in \mathbb{R}^{+}$.

We deduce from (10) and (11) that for each $t \in \mathbb{R}^{+}$:

$$
\lim _{n \rightarrow \infty} F\left(t+n \omega_{1}, s\right)=G(t, s)
$$

uniformly for $s \in \mathbb{R}^{+}$.

In a similar way, we can show there exists a function $H$ such that for each $s \in \mathbb{R}^{+}$:

$$
\lim _{n \rightarrow \infty} F\left(t, s+n \omega_{2}\right)=H(t, s)
$$

uniformly for $t \in \mathbb{R}^{+}$.

Definition 5. A function $x \in C_{b}\left(\mathbb{R}^{+}, X\right)$ is said to be a mild solution of Problem (9) if:

$$
x(t)=T(t) x_{0}+\int_{0}^{t} T(t-s) F(s, x(s)) d s, t \in \mathbb{R}^{+},
$$

where $(T(t))_{t \geq 0}$ is an exponentially stable $C_{0}$-semigroup.

Now, we can establish the following theorem.

Theorem 4. Let $F: \mathbb{R}^{+} \times X \rightarrow X$ be the $\left(\omega_{1}, \omega_{2}\right)$-quasi-periodic limit uniformly for $x$ in bounded subsets of $X$, and assume $F(t, x)=f(t, t, x)$, where $f \in P L_{\left(\omega_{1}, \omega_{2}\right)}\left(\mathbb{R}^{+} \times \mathbb{R}^{+} \times X, X\right)$. Assume that $f$ satisfies a Lipschitz condition in $x$ uniformly in $t, s \in \mathbb{R}^{+}$:

$$
\|f(t, s, x)-f(t, s, y)\| \leq L\|x-y\|
$$

for all $x, y \in X$ and $t, s \in \mathbb{R}^{+}$, where $L>0$. If $M L<r$, then there exists an asymptotically quasi-periodic mild solution of Problem (9).

Proof. Define the function $T: \mathbb{R}^{+} \times \mathbb{R}^{+} \rightarrow X$ by:

$$
T(x, y)=\left\{\begin{array}{l}
T(y) x_{0}, y \geq x \\
T(x) x_{0}, x \geq y
\end{array}\right.
$$


Then, we can define the operator $\Gamma$ on the space $A Q P_{\left(\omega_{1}, \omega_{2}\right)}\left(\mathbb{R}^{+} \times \mathbb{R}^{+}, X\right)$ by:

$$
\Gamma \varphi(x, y)=T(x, y)+\left\{\begin{array}{l}
\int_{0}^{x} T(x-s) f(s, s+y-x, \varphi(s, s+y-x)) d s, y \geq x, \\
\int_{0}^{y} T(y-s) f(s+x-y, s, \varphi(s+x-y, s)) d s, x \geq y,
\end{array}\right.
$$

where $\varphi \in A Q P_{\left(\omega_{1}, \omega_{2}\right)}\left(\mathbb{R}^{+} \times \mathbb{R}^{+}, X\right) \subset P L_{\left(\omega_{1}, \omega_{2}\right)}\left(\mathbb{R}^{+} \times \mathbb{R}^{+}, X\right)$.

Clearly, $T(x, y) \in A Q P_{\left(\omega_{1}, \omega_{2}\right)}\left(\mathbb{R}^{+} \times \mathbb{R}^{+}, X\right)$. By Lemma 1 and Theorem 3, one has $\Gamma \varphi \in$ $A Q P\left(\omega_{1}, \omega_{2}\right)\left(\mathbb{R}^{+} \times \mathbb{R}^{+}, X\right)$. Note that the space $A Q P_{\left(\omega_{1}, \omega_{2}\right)}\left(\mathbb{R}^{+} \times \mathbb{R}^{+}, X\right)$ is a Banach space by Theorem 1. Finally, for $\varphi_{1}, \varphi_{2} \in A Q P_{\left(\omega_{1}, \omega_{2}\right)}\left(\mathbb{R}^{+} \times \mathbb{R}^{+}, X\right)$, one has:

$$
\left\|\Gamma \varphi_{1}-\Gamma \varphi_{2}\right\|_{\infty} \leq \frac{M L}{r}\left\|\varphi_{1}-\varphi_{2}\right\|_{\infty}
$$

which shows that $\Gamma$ is a contraction. By the contraction mapping principle, there exists a unique $\bar{\varphi}(x, y) \in A Q P_{\left(\omega_{1}, \omega_{2}\right)}\left(\mathbb{R}^{+} \times \mathbb{R}^{+}, X\right)$ such that $\Gamma \bar{\varphi}(x, y)=\bar{\varphi}(x, y)$, that is:

$$
\bar{\varphi}(x, y)=T(x, y)+\left\{\begin{array}{l}
\int_{0}^{x} T(x-s) f(s, s+y-x, \bar{\varphi}(s, s+y-x)) d s, y \geq x \\
\int_{0}^{y} T(y-s) f(s+x-y, s, \bar{\varphi}(s+x-y, s)) d s, x \geq y .
\end{array}\right.
$$

If we denote $\bar{\Phi}(t)=\bar{\varphi}(t, t)$, then we have $\Gamma \bar{\Phi}(t)=\bar{\Phi}(t)$, that is:

$$
\bar{\Phi}(t)=T(t) x_{0}+\int_{0}^{t} T(t-s) F(s, \bar{\Phi}(s)) d s .
$$

Therefore, $\bar{\Phi}$ is a solution of Problem (9). Moreover, $\bar{\Phi}$ is asymptotically quasi-periodic by Proposition 8.

Remark 3. The operator $\Gamma$ in Theorem 4 may be constructed in a different way, so we cannot show the uniqueness of the solution of Problem (9).

Example 4. Consider the following problem:

$$
\left\{\begin{array}{l}
\frac{\partial}{\partial t} u(t, x)=\frac{\partial^{2}}{\partial x^{2}} u(t, x)+A(t) f(u(t, x)), t \in \mathbb{R}^{+}, x \in[0, \pi] \\
u(t, 0)=u(t, \pi)=0, t \in \mathbb{R}^{+} \\
u(0, x)=g(x), x \in[0, \pi]
\end{array}\right.
$$

where the functions $g:[0, \pi] \rightarrow \mathbb{R}$ and $f: \mathbb{R} \rightarrow \mathbb{R}$ are appropriate bounded continuous functions and $A$ defined as $A(t)=a(t, t)$, where $a \in P L_{\left(\omega_{1}, \omega_{2}\right)}\left(\mathbb{R}^{+} \times \mathbb{R}^{+}, \mathbb{R}\right)$. Besides, $f$ satisfies:

$$
|f(x)-f(y)| \leq L|x-y|, x, y \in \mathbb{R},
$$

where $L>0$. Let $X=L^{2}([0, \pi])$, and let $A$ be the operator given by $A u=u^{\prime \prime}$ with domain $D(A)=\{u \in$ $\left.X: u^{\prime \prime} \in X, u(0)=u(\pi)=0\right\}$. Clearly, $A$ is the infinitesimal generator of an analytic semigroup $(T(t))_{t \geq 0}$ on $X$. Moreover, $A$ has a discrete spectrum with eigenvalues $-n^{2}, n \in \mathbb{N}$, and corresponding normalized eigenfunctions given by $z_{n}(\xi)=\left(\frac{2}{\pi}\right)^{\frac{1}{2}} \sin (n \xi)$. Furthermore, $\left\{z_{n}: n \in \mathbb{N}\right\}$ is an orthonormal basis of $X$ and $T(t) x=\sum_{n=1}^{\infty} e^{-n^{2} t}\left\langle x, z_{n}\right\rangle z_{n}$ for $x \in X$. Therefore, one has $\|T(t)\| \leq e^{-t}, t \in \mathbb{R}^{+}$. Therefore, if $\|a\|_{\infty} L<1$, (12) has an asymptotically quasi-periodic mild solution by Theorem 4. 


\section{Some Remarks and Questions}

Proposition 6 and Proposition 8 make sure that these quasi-periodic limit functions can be regarded as a generalization of asymptotically quasi-periodic functions. Here, a question arises: Could we generalize quasi-periodic limit functions again?

It seems more general if we define $F(t)=f(t, t)$ without $\mathrm{H} 1$ and $\mathrm{H} 2$ in Definition 2. However, we have the following example.

Example 5. Define:

$$
f(x, y)= \begin{cases}f_{1}(x, y), & y \geq k_{1} x \\ f_{2}(x, y), & k_{2} x<y<k_{1} x \\ f_{3}(x, y), & y \leq k_{2} x\end{cases}
$$

where $x, y \in \mathbb{R}^{+}, k_{1}>1,0<k_{2}<1$. $f_{1}$ is a continuous function such that $\lim _{n \rightarrow \infty} f_{1}\left(x, y+n \omega_{2}\right)=$ $g_{1}(x, y) \cdot f_{3}$ is a continuous function such that $\lim _{n \rightarrow \infty} f_{3}\left(x+n \omega_{1}, y\right)=h_{3}(x, y) \cdot f_{1}(0,0)=f_{3}(0,0) . f_{2}$ is an arbitrary continuous function such that $f$ is continuous on $\mathbb{R}^{+} \times \mathbb{R}^{+}$. Then, $f$ is a $\left(\omega_{1}, \omega_{2}\right)$-periodic limit function. However, $F(t)=f(t, t)=f_{2}(t, t)\left(t \geq t_{0}, t_{0}>0\right)$ can be an arbitrary continuous function.

It is interesting to ask whether there is another way to generalize asymptotically quasi-periodic functions without using a function with several variables.

Let $x \in C_{b}\left(\mathbb{R}^{+}, X\right)$. If for every $\varepsilon>0$, there exist $f_{i} \in P_{\omega_{1}} L\left(\mathbb{R}^{+}, \mathbb{C}\right), g_{i} \in P_{\omega_{2}} L\left(\mathbb{R}^{+}, \mathbb{C}\right), F_{i} \in$ $P_{\omega_{2}} L\left(\mathbb{R}^{+}, X\right)$ and $G_{i} \in P_{\omega_{1}} L\left(\mathbb{R}^{+}, X\right)(i=1,2, \cdots, N)$ such that:

$$
\left\|x-\sum_{i=1}^{N}\left(f_{i} F_{i}+g_{i} G_{i}\right)\right\|<\varepsilon
$$

then denote the set of $x$ by $\overline{Q P L}_{\left(\omega_{1}, \omega_{2}\right)}\left(\mathbb{R}^{+}, X\right)$.

Note that $\sum_{i=1}^{N}\left(f_{i} F_{i}+g_{i} G_{i}\right) \in Q P L_{\left(\omega_{1}, \omega_{2}\right)}\left(\mathbb{R}^{+}, X\right)$ for each $N \in \mathbb{N}$. Therefore, there is a natural question as follows.

Question 4.2: Does $\overline{Q P L}_{\left(\omega_{1}, \omega_{2}\right)}\left(\mathbb{R}^{+}, X\right)$ equal $Q P L_{\left(\omega_{1}, \omega_{2}\right)}\left(\mathbb{R}^{+}, X\right)$ ?

Author Contributions: Investigation, R.X., Z.X. and J.L. All authors contributed equally to the manuscript and typed, read, and approved the final manuscript.

Funding: The work of the first author is supported by the research project of Tianjin Municipal Education Commission (Grant No. 160022), the Doctoral Scientific Research Foundation of Tianjin University of Commerce (Grant No. R160101), and the National Nurture Fund of Tianjin University of Commerce (Grant No. 170113). The research of the second author is supported by the National Natural Science Foundation of China (Grant No. 11501507) and the Natural Science Foundation of Zhejiang Province (Grant No. LY19A010013). The work of the third author is supported by the Natural Science Foundation of Hebei Province (Grant No. A2016203341).

Conflicts of Interest: The authors declare no conflict of interest.

\section{References}

1. Bohl, P. Über eine Differentialgleichung der Störungstheorie. J. Reine Angew. Math. 1906, 131, $231-268$.

2. Hagihara, Y. Piers Bohls Work on Celestial Mechanics; Smithsonian Institution, Astrophysical Observatory: Cambridge, MA, USA, 1967.

3. Besicovitch, A.S. Almost Periodic Functions; Cambridge University Press: Cambridge, UK, 1932.

4. Kuksin, S. Hamiltonian perturbations of infinite-dimensional linear systems with imaginary spectrum. Funct. Anal. Its Appl. 1987, 21, 192-205.

5. Wayne, E. Periodic and quasi-periodic solutions of nonlinear wave equations via KAM theory. Commun. Math. Phys. 1990, 127, 479-528.

6. Bourgain, J. Quasi-periodic solutions of Hamiltonian perturbations of 2D linear Schrödinger equations. Ann. Math. 1998, 148, 363-439. 
7. Pöschel, J. A KAM-theorem for some nonlinear partial differential equations. Annali Scuola Normale Superiore di Pisa-Classe di Scienze 1996, 23, 119-148.

8. Geng, J.; Xu, X.; You, J. An infinite dimensional KAM theorem and its application to the two dimensional cubic Schrödinger equation. Adv. Math. 2011, 226, 5361-5402.

9. Procesi, C.; Procesi, M. A normal form for the Schrödinger equation with analytic non-linearities. Commun. Math. Phys. 2012, 312, 501-557.

10. Mi, L.; Zhang, K. Quasi-periodic solutions for perturbed generalized KdV equation. Nonlinear Anal. 2016, 32, 314-337. [CrossRef]

11. Zhang, M. Quasi-periodic solutions of two dimensional Schrödinger equations with Quasi-periodic forcing. Nonlinear Anal. 2016, 135, 1-34. [CrossRef]

12. Giuliani, F. Quasi-periodic solutions for quasi-linear generalized KdV equations. J. Differ. Equ. 2017, 262, 5052-5132. [CrossRef]

13. Baldi, P.; Berti, M.; Montalto, R. KAM for autonomous quasi-linear perturbations of KdV. Ann. Inst. Henri Poincaré Anal. Non Linéaire 2016, 33, 1589-1638. [CrossRef]

14. Lou, Z.; Geng, J. Quasi-periodic response solutions in forced reversible systems with Liouvillian frequencies. J. Differ. Equ. 2017, 263, 3894-3927.

15. Chen, B.; Gao, Y.; Jiang, S.; Li, Y. Quasi-periodic solutions to nonlinear beam equations on compact Lie groups with a multiplicative potential. J. Differ. Equ. 2018, 264, 6959-6993. [CrossRef]

16. Wang, Y. Quasi-periodic solutions for a completely resonant beam equation with a nonlinear term depending on the time and space variables. Nonlinear Anal. 2019, 189, 111585. [CrossRef]

17. Shi, Y.; Xu, J. Quasi-periodic solutions for a class of beam equation system. Discret. Cont. Dyn. B 2020, 25, 31-53. [CrossRef]

18. Küpper, T.; Yuan, R. On quasi-periodic solutions of differential equations with piecewise constant argument. J. Math. Anal. Appl. 2002, 267, 173-193. [CrossRef]

19. Shi, G.; Yan, D. Quasi-periodic solutions for nonlinear wave equation with singular Legendre potential. Bound. Value Probl. 2019, 1, 108. [CrossRef]

20. Ragusa, M.A. Cauchy-Dirichlet problem associated to divergence form parabolic equations. Commun. Contemp. Math. 2004, 6, 377-393. [CrossRef]

21. Xie, R.; Zhang, C. Space of $\omega$-periodic limit functions and its applications to an abstract Cauchy problem. J. Funct. Spaces 2015, 2015, 953540. [CrossRef]

22. Manou-Abi, S.M.; Dimbour, W. Asymptotically periodic solution of a stochastic differential equation. Bull. Malays. Math. Sci. Soc. 2019, 1-29. [CrossRef]

23. Dimbour, W.; Mawaki, S. Asymptotically $\omega$-periodic solutions for an evolution differential equation via $\omega$-periodic limit functions. J. Pure Appl. Math. 2017, 113, 59-71. [CrossRef] 